\title{
Journal of Neurotrauma
}

Journal of Neurotrauma: http://mc.manuscriptcentral.com/neurotrauma

\section{Serum metabolites associate with CT findings following TBI}

\begin{tabular}{|c|c|}
\hline Journal: & Journal of Neurotrauma \\
\hline Manuscript ID & NEU-2017-5272.R2 \\
\hline Manuscript Type: & Regular Manuscript \\
\hline Date Submitted by the Author: & 19-Apr-2018 \\
\hline Complete List of Authors: & $\begin{array}{l}\text { Dickens, Alex; University of Turku, Turku Centre for Biotechnology } \\
\text { Posti, Jussi; Turku University Hospital, Division of Clinical Neurosciences, } \\
\text { Department of Rehabilitation and Brain Trauma; University of Turku, } \\
\text { Department of Neurology; Turku University Hospital, Division of Clinical } \\
\text { Neurosciences, Department of Neurosurgery } \\
\text { Takala, Riikka; Turku University Hospital, Perioperative Services, Intensive } \\
\text { Care Medicine and Pain Management } \\
\text { Ala-Seppälä, Henna; University of Turku, Department of Neurology } \\
\text { Mattila, Ismo; Steno Diabetes Center AS } \\
\text { Coles, Jonathan Coles; University of Cambridge, Department of Clinical } \\
\text { Neurosciences } \\
\text { Frantzen, Janek; Turku University Hospital, Division of Clinical } \\
\text { Neurosciences, Department of Rehabilitation and Brain Trauma; University } \\
\text { of Turku, Department of Neurology; Turku University Hospital, Division of } \\
\text { Clinical Neurosciences, Department of Neurosurgery } \\
\text { Hutchinson, Peter; University of Cambridge, Department of Clinical } \\
\text { Neurosciences } \\
\text { Katila, Ari; Turku University Hospital, Perioperative Services, Intensive } \\
\text { Care Medicine and Pain Management } \\
\text { Kyllönen, Anna; University of Turku, Department of Neurology } \\
\text { Maanpää, Henna-Riikka; Turku University Hospital, Division of Clinical } \\
\text { Neurosciences } \\
\text { Newcombe, Virginia; University of Cambridge, Division of Anaesthesia } \\
\text { Outtrim, Joanne; University of Cambridge, Division of Anaesthesia } \\
\text { Tallus, Jussi; University of Turku, Centre for Cognitive Neuroscience } \\
\text { Carpenter, Keri; University of Cambridge, Devision of Neurosurgery, } \\
\text { Department of Clinical Neurosciences } \\
\text { Menon, David; University of Cambridge, Head, Division of Anaesthesia } \\
\text { Hyotylainen, Tuulia; Örebro University, Department of Chemistry } \\
\text { Tenovuo, Olli; Turku University Hospital, Division of Clinical Neurosciences, } \\
\text { Department of Neurosurgery; University of Turku, Department of } \\
\text { Neurology } \\
\text { Oresic, Matej; University of Turku, Turku Centre for Biotechnology; } \\
\text { University of Örebro, School of Medical Science }\end{array}$ \\
\hline Keywords: & $\begin{array}{l}\text { METABOLISM, TRAUMATIC BRAIN INJURY, CT SCANNING, HUMAN } \\
\text { STUDIES, BIOMARKERS }\end{array}$ \\
\hline $\begin{array}{r}\text { Manuscript Keywords (Search } \\
\text { Terms): }\end{array}$ & Metabolomics, Traumatic Brain Injury, CT, Biomarkers \\
\hline
\end{tabular}




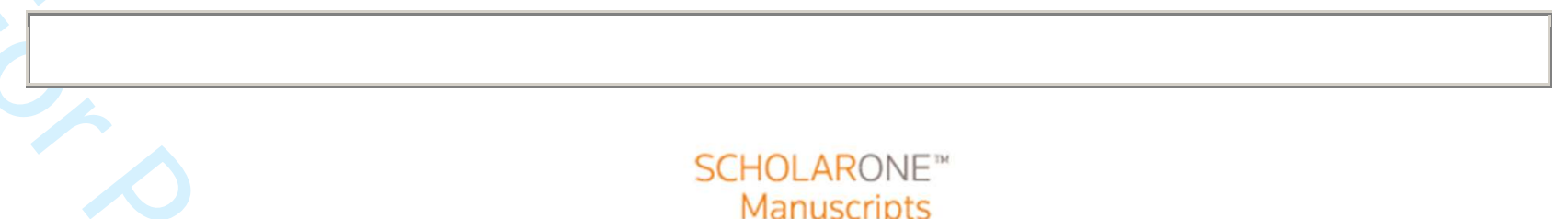

10 11 12 13 14 15 16 17 8 


\section{Serum metabolites associate with CT findings following TBI}

Running head: Serum metabolomics associates with CT imaging in TBI

Authors: Alex M. Dickens, D.Phil. ${ }^{1 *}$, Jussi P. Posti, MD, PhD ${ }^{2,3,4}$, Riikka S.K. Takala, MD, PhD ${ }^{5}$, Henna Ala-Seppälä, $\mathrm{MD}^{3}$, Ismo Mattila ${ }^{6}, \mathrm{MSc}$, Jonathan P. Coles, MD, $\mathrm{PhD}^{7}$, Janek Frantzén, MD, $\mathrm{PhD}^{2,3,4}$, Peter J. Hutchinson, FRCS (SN), $\mathrm{PhD}^{8}$, Ari J. Katila, $\mathrm{MD}^{5}$, Anna Kyllönen, $\mathrm{MD}^{3}$, Henna-Riikka Maanpää, $\mathrm{MD}^{3}$, Virginia Newcombe, $\mathrm{MD}, \mathrm{PhD}^{7}$, Joanne Outtrim, $\mathrm{RN}^{7}$, Jussi Tallus, $\mathrm{MD}^{3}$, Keri L H Carpenter, $\mathrm{PhD}^{8}$, David K. Menon, MD, $\mathrm{PhD}^{7}$, Tuulia Hyötyläinen, $\mathrm{PhD}^{9}$, Olli Tenovuo, $\mathrm{MD}, \mathrm{PhD}^{2,3}$, and Matej Orešič, $\mathrm{PhD}^{1,10}$.

*These authors contributed equally to this work.

Affiliations: ${ }^{1}$ Turku Centre for Biotechnology, University of Turku, Finland; ${ }^{2}$ Turku Brain Injury Centre, Turku University Hospital, Finland; ${ }^{3}$ Department of Neurology, University of Turku, Finland; ${ }^{4}$ Division of Clinical Neurosciences, Department of Neurosurgery, Turku University Hospital, Finland; ${ }^{5}$ Perioperative Services, Intensive Care Medicine and Pain Management, Turku University Hospital and University of Turku, Finland, ${ }^{6}$ Steno Diabetes Center Copenhagen, DK-2820 Gentofte, Denmark. ${ }^{7}$ Division of Anaesthesia, Department of Medicine, University of Cambridge, Addenbrooke's Hospital, Cambridge, United Kingdom; ${ }^{8}$ Division of Neurosurgery, Department of Clinical Neurosciences, University of Cambridge, Addenbrooke's Hospital, Cambridge, United Kingdom, ${ }^{9}$ Department of Chemistry, Örebro University, Örebro, Sweden, and ${ }^{10}$ Schools of Medical Science, Örebro University, Örebro, Sweden.

Co-first and Corresponding Author: Alex M. Dickens

Turku Centre for Biotechnology

Tykistökatu 6

20520 Turku

FINLAND

Email: alex.dickens@utu.fi, Phone: +358294503798 Fax: +358 294505040

Mailing Addresses of all other authors:

Jussi P Posti

Division of Clinical Neurosciences, Department of Neurosurgery

Turku University Hospital

20521Turku

Finland

Email: Jussi.Posti@utu.fi, Phone: +358 2333 8585, Fax: +358 23133052

Riikka S.K. Takala 
Perioperative Services, Intensive Care Medicine and Pain

PO Box 52,

29521 Turku

Finland

Email: riikka.takala@gmail.com, Phone: +3582 3338585 Fax: +358 23133960

Henna Ala-Seppälä

Department of Neurology

University of Turku

20014 Turku

Finland

Email: hmalse@utu.fi, Phone: +3582 333 8585, Fax: +358 294505040

Ismo Mattila

Steno Diabetes Center Copenhagen

Systems Medicine

Niels Steensensvej 2

DK-2820 Gentofte

Email: ismo.matias.mattila@,regionh.dk, Phone: + 45309133 43, Fax: +358 294505040

Jonathan P. Coles

Division of Anaesthesia

Department of Medicine

University of Cambridge

Addenbrooke's Hospital

Cambridge

United Kingdom

CB2 2QQ

Email: jpc44@wbic.cam.ac.uk, Phone: +44 1223 336946, Fax: +44 1223217887

Janek Frantzén

Division of Clinical Neurosciences, Department of Neurosurgery

Turku University Hospital

20521Turku

Finland

Email: Janek.frantzen@tyks.fi, Phone: +358 2 3130617, Fax: +358 23133052

Peter J. Hutchinson

Division of Neurosurgery

Department of Clinical Neurosciences

University of Cambridge

Box 167, Cambridge Biomedical Campus

Cambridge

CB2 0QQ

UK

Email:pjah2@,cam.ac.uk, Phone: +44 1223 336946, Fax: +44 1223216926

Ari J. Katila

Perioperative Services, Intensive Care Medicine and Pain 
PO Box 52, 29521 Turku

Finland

Email: ari.katila@tyks.fi, Phone: +35823338585 Fax: +35823133960

Anna Kyllönen

Department of Neurology

University of Turku

20014 Turku

Finland

Email: akkyll@utu.fi, Phone: +358 2333 8585, Fax: +358 294505040

Henna-Riikka Maanpää

Department of Neurology

University of Turku

20014 Turku

Finland

Email: hrmaan@utu.fi, Phone: +358 2333 8585, Fax: +358 294505040

Virginia Newcombe

Division of Anaesthesia

Department of Medicine

University of Cambridge

Addenbrooke's Hospital

Cambridge

United Kingdom

CB2 2QQ

Email: vfjn2@cam.ac.uk, Phone: +44 1223 336946, Fax: +44 1223217887

Joanne Outtrim

Division of Anaesthesia

Department of Medicine

University of Cambridge

Addenbrooke's Hospital

Cambridge

United Kingdom

CB2 2QQ

Email: jgo22@cam.ac.uk, Phone: +44 1223 336946, Fax: +44 1223217887

Jussi Tallus

Department of Neurology

University of Turku

20014 Turku

Finland

Email: jptall@utu.fi, Phone: +358 2333 8585, Fax: +358 294505040

Keri L H Carpenter

Division of Neurosurgery

Department of Clinical Neurosciences

University of Cambridge 
Box 167, Cambridge Biomedical Campus

Cambridge

CB2 0QQ

UK

Email: klc1000@,wbic.cam.ac.uk, Phone: +44 1223 746453, Fax: +44 1223216926

David K. Menon

Division of Anaesthesia

Department of Medicine

University of Cambridge

Addenbrooke's Hospital

Cambridge

United Kingdom

CB2 2QQ

Email: dkm13@wbic.cam.ac.uk, Phone: +44 1223 217891, Fax: +44 1223217887

Tuulia Hyötyläinen

Department of Chemistry

University of Örebro

70285 Örebro

Sweden

Email: Tuulia.Hyotylainen@oru.se Phone: +46 19 303487, Fax: +358 294505040

Olli Tenovuo

Turku University Hospital

Kiinamyllynkatu 4-8

20520 Turku, Finland

Email: ollten@utu.fi, Phone: +358 50 4383802, Fax: +35823132737

Matej Orešič

Turku Centre for Biotechnology

Tykistökatu 6

FI-20520 Turku

FINLAND

Email: matej.oresic@utu.fi Phone: +358 44972 6094, Fax: +358 294505040 


\begin{abstract}
There is a need to rapidly detect patients with traumatic brain injury (TBI) who require head computed tomography (CT). Given the energy crisis in the brain following TBI, we hypothesized that serum metabolomics would be a useful tool for developing a set of biomarkers to determine the need for $\mathrm{CT}$ and to distinguish between different types of injuries observed.
\end{abstract}

Logistic regression models using metabolite data from the discovery cohort $(n=144$, Turku, Finland) were used to distinguish between patients with traumatic intracranial findings and negative findings on head CT. The resultant models were then tested in the validation cohort ( $n=66$, Cambridge, UK). The levels of glial fibrillary acidic protein and ubiquitin C-terminal hydrolase-L1 were also quantified in the serum from the same patients.

Despite there being significant differences in the protein biomarkers in patients with TBI, the model that determined the need for a $\mathrm{CT}$ scan validated poorly (AUC=0.64: Cambridge patients). However, using a combination of six metabolites (two amino acids, three sugar derivatives and one ketoacid) it was possible to discriminate patients with intracranial abnormalities on $\mathrm{CT}$ and patients with a normal $\mathrm{CT}(\mathrm{AUC}=0.77$ in Turku patients and $\mathrm{AUC}=0.73$ in Cambridge patients). Furthermore, a combination of three metabolites could distinguish between diffuse brain injuries and mass lesions (AUC $=0.87$ in Turku patients and $\mathrm{AUC}=0.68$ in Cambridge patients).

This study identifies a set of validated serum polar metabolites, which associate with the need for a CT scan. Additionally, serum metabolites can also predict the nature of the brain injury. These metabolite markers may prevent unnecessary CT scans, thus reducing the cost of diagnostics and radiation load.

Key Words: Metabolism, Traumatic Brain Injury, CT Scanning, Human Studies, Biomarkers 


\section{Introduction}

The diagnosis of traumatic brain injury (TBI) in the emergency room often relies upon a clinical neurological exam and neuroimaging. (1) The ability to more rapidly predict which patients will require $\mathrm{CT}$ imaging following a TBI is a long-standing problem and remains a key goal in the ability to triage patients upon admission. (2)

Previous efforts have focused on quantifying proteins such as S100 calcium-binding protein $\beta$ (S100ß), (3) glial fibrillary acidic protein (GFAP), (4) and ubiquitin C-terminal hydrolase-L1 (UCH-L1) (5) in the blood. Whilst studies have demonstrated the ability of these proteins to generate models which can predict the need for CT scan with reasonable areas under the curves (AUC $=0.78 \mathrm{~S} 100 \beta$, (6) 0.84 GFAP, (6) $0.73 \mathrm{UCH}-\mathrm{L} 1$ (5)), the authors have not demonstrated the predictive power of these models using independent data sets. S100 $\beta$ is probably the most studied protein biomarker for TBI. However, it is not specific to brain or TBI $(7,8)$ : the levels of circulating S100 $\beta$ have been shown to vary after exercise (9) and patients who have sustained polytrauma where adipose tissue and bone are affected are known to exhibit high levels of S100ß. $(10,11)$ Recently however, GFAP, a protein expressed in the astrocytes within the CNS (12) has been shown to be a better predictor of the need for CT imaging. However, these studies did not contain an independent validation cohort, which is an essential component when employing diagnostic or prognostic models due to the risk of overfitting of the predictive model if only one dataset is used (13). Additionally, these proteins appear to have better predictive power in patients with more severe TBI $(1,14,15)$. This observation could well reflect the fact that changes in these proteins happen in the CNS behind the blood-brain-barrier (BBB) or, in the case of GFAP changes in the BBB itself, which will become more permeable in more severe cases $(16,17)$, thus altering the levels of brain derived proteins in the blood. Furthermore, GFAP may well also be non-specific to TBI, because Schwann cells in the periphery are known to increase 
GFAP production following injury $(18,19)$. Indeed, our recent study showed that GFAP and UCH-L1 were unable to separate patients with CT-negative mild TBI (mTBI) from patients with orthopaedic injuries. (20)

To date metabolic studies in TBI have mainly focused on trying to measure the changes in metabolism in the brain using a combination of techniques such as Magnetic Resonance Spectroscopy (MRS), Positron Emission Tomography (PET), and measuring polar metabolites from brain microdialysates. (21) These techniques are expensive and/or invasive and not widely available to all TBI patients especially those with less severe injuries. Previous attempts at looking at metabolic changes in the blood have targeted a very small number of molecules such as glucose and lactate. (22) To date the application of modern untargeted metabolomic approaches to study TBI have been sparse and to our knowledge there are no studies exploring the role of metabolomics in detecting the need for a CT scan following a TBI. (23) Serum metabolomics is a powerful tool for non-invasively identifying biomarkers in disease. (24-26) We have previously shown that the severity of TBI and the eventual outcomes are associated with the changes in serum metabolome. (21) Furthermore, attempts at generating diagnostic models in animal models of TBI (27) have been reported using a combination of molecular lipids and polar metabolites measured from serum. However, most of these studies lacked a suitable validation cohort and therefore, their use as a diagnostic tool is currently still limited. Here we use gas chromatography, which covers small polar metabolites such as sugars, amino acids and free fatty acids in an untargeted approach $(28,29)$ to screen for new metabolites which can predict the need for a CT following a TBI.

The principal aim of this study was to assess whether serum metabolomics can be used distinguish between patients with traumatic intracranial findings and negative findings on head CT using logistic regression modelling. We then compared the metabolomics models 
to the results from protein assays acquired from the blood and explored if together they have an additive effect in terms of predicting the need for a head CT. In addition to this primary aim, we also assessed if serum metabolomics can distinguish between diffuse CT pathology and mass lesions. Finally, we explore the potential origins of the metabolite changes by comparing the metabolite changes in serum to that of metabolite changes in the cerebral microdialysis of the same individuals and comparing it to an appropriate control group. 


\section{Methods}

\section{Ethics Statement}

All protocols used in this study were approved by South-West Finland Hospital District Research Ethics Committee, the Cambridgeshire 2 Research Ethics Committee, and the Norfolk Research Ethics Committee depending which country the samples were collected in. Oral and written consent was obtained from either the patient or their next of kin. All patients received treatment based on local standards and current international guidelines and recommendations. (30)

Sample selection and clinical details

The patients in this study were a subgroup of individuals from our previously published cohort. (21). The patients were recruited as part of the EU funded TBI care (Evidence-based Diagnostic and Treatment Planning Solution for Traumatic Brain Injuries) project. Patients were included if they were older than 18 years (16 in the UK) and had a clinical diagnosis of TBI and indications on a head CT according to the National Institute for Health and Care Excellence (NICE) criteria. Patients were excluded if the injuries were blast-induced or penetrating injury, chronic subdural hematoma, pre-existing brain injuries or conditions, which caused non-independent living, TBI or suspected TBI two weeks prior to recruitment, non-native speaker, and no if no consent was obtained.

Out of 620 screened patients with suspected TBI, total 203 adults with acute TBI and 40 patients with orthopedic injuries with no known head trauma were recruited at Turku University Hospital (Turku, Finland). With serum samples available for comprehensive metabolomics profiling, 144 patients with verified TBI and 28 patients with orthopedic injury and carefully excluded TBI were included in the discovery cohort. The causes for not being recruited among patients with suspected TBI was available for 568 patients and the 
commonest reasons for exclusion were: research staff was unable to recruit $(n=191,34 \%)$, age less than 18 years $(n=82,14 \%)$, and uncertain diagnosis for TBI $(n=75,13 \%)$.

Out of 1122 screened patients with suspected TBI, altogether 188 adults with acute TBI and 41 orthopedic controls were recruited from Addenbrooke's Hospital (Cambridge, UK). With serum samples available for comprehensive metabolomics profiling, 188 patients with verified TBI and 27 patients with orthopedic injury and rigorously excluded TBI were included in the validation cohort. The commonest reasons for exclusion among patients with suspected TBI were: research staff was unable to recruit $(n=352,31 \%)$, patient being too frail for reliable follow-up $(n=275,25 \%)$, and pre-existing disease $(n=219,20 \%)$.

The patient demographic data is summarized in Table 1.

The blood samples used in this analysis was collected within $12 \mathrm{~h}$ of admission to hospital by research nurses and other study personnel. There are some patients who were found unconscious and transferred to hospital and patients who sustained mTBI and sought for medical attention with latency. In these patients, the exact time of injury is unknown. The serum was allowed to clot for $30 \mathrm{mins}$ at $4{ }^{\circ} \mathrm{C}$. The blood was then centrifuged $(10 \mathrm{~min}$, $10,000 \mathrm{rpm}, 4^{\circ} \mathrm{C}$ ). All blood samples were stored at $-70^{\circ} \mathrm{C}$ in aliquots to prevent the need for freeze-thaw cycles. Cerebral microdialysis were collected from a subsection of the individuals in Cambridge $(\mathrm{n}=12)$ who had had a sTBI. The samples were collected using a CMA71, 100 kDa molecular weight cut-off catheter (M Dialysis AB, Stockhom, Sweden) via a cranial device (Technicam, Newton Abbot, UK) with a perfustion rate of $0.3 \mathrm{ml} / \mathrm{min}$ using a CMA106 pump (M Dialysis AB). The fluid consisted of CNS perfusion fluid (M Dialysis AB). The CT images were graded using the Marshall classification (31) and this was used as a classifier for the aims in this study:

Aim 1: Marshall Grade 1 (CT negative) vs. Marshall Grade 2-6 (CT positive) 
Aim 2: Marshall Grade 2-4 (diffuse injury) vs. Marshall Grade 5-6 (mass lesion)

Marshall classification was chosen, because it can be appropriately used for the aforementioned patient group division and to address the clinical questions. The CT scans were analyzed by neuroradiologists and double-read by a senior neurosurgeon (JPP) and a neurologist (OT).

The samples were collected and processed as previously described. (21)

\section{Metabolomics}

Metabolomic analysis was carried out with comprehensive two-dimensional gas chromatography (Agilent 6890, Agilent Technologies, Santa Clara, CA) combined with timeof flight mass spectrometry (Pegasus GC-HRT, Leco Corp., St. Joesph, MI, USA) as described. (21) Briefly, $10 \mu \mathrm{l}$ of internal standards (C17:0 186.5 mg/L, deuterated valine (37 $\mathrm{mg} / \mathrm{L}$, and succinic acid-d4 (63 mg/l)) were added to serum samples $(30 \mu \mathrm{l})$ or microdialysate samples $(100 \mu \mathrm{l})$. The protein was precipitated by the addition of $400 \mu \mathrm{l}$ of $\mathrm{MeOH}$ and the resultant mixture was vortexed for 2 mins. The samples were then centrifuged (7800 g, $5 \mathrm{~min}$, room temperature) prior to being left to settle $\left(30 \mathrm{mins},-20^{\circ} \mathrm{C}\right)$. The supernatant was then removed and evaporated to dryness under a flow of $\mathrm{N}_{2}$ gas at $45{ }^{\circ} \mathrm{C}$. The metabolites underwent a two-step derivatization, initially methoxyamine hydrochloride ( $98 \%$ in pyridine) was added and the resultant mixture was incubated for $1 \mathrm{~h}$ at $45^{\circ} \mathrm{C}$. Next N-methyl-Ntrimethylsilyltrifluoroacetamide $(25 \mu \mathrm{L})$ was added and the sample incubated for a further $1 \mathrm{~h}$ at $45{ }^{\circ} \mathrm{C}$. Just prior to injection a series of alkane standards (n-alkanes, $25 \mu \mathrm{L}, \mathrm{c}=8 \mathrm{mg} / \mathrm{l}$ ) and an injection standard (4,4'-dibromooctafluorobiphenyl, $50 \mu \mathrm{l}, \mathrm{c}=10 \mathrm{mg} / \mathrm{l})$ were added in order to calculate the retention index of each metabolite. The derivatization and sample injection was automated by using a multipurpose sampler (Gerstel, Mülheim an der Ruhr, Germany). The samples were separated using a $10 \mathrm{~m}$ x $0.18 \mathrm{~mm}$ inner diameter (ID) Rxi-5 
ms (Resteck Corp., Bellefonte, PA, USA) column with a film thickness of $0.18 \mu \mathrm{m}$ as a first column and a $1.5 \mathrm{~m}$ x $0.1 \mathrm{~mm}$ ID BPX-50 (SGE Analytical Science Austin, TX, USA) column with film thickness of $0.1 \mu \mathrm{m}$ as a second column. A deactivated retention gap column (phenyl methyl, $1.5 \mathrm{~m} \times 0.53 \mathrm{~mm}$ ID) was used as a guard column. $1 \mu \mathrm{l}$ of samples was injected in splitless mode $\left(240^{\circ} \mathrm{C}\right)$. The splitless mode lasted for $90 \mathrm{~s}$ with high purity helium (Aga, Espoo, Finland) used as the carrier gas. Constant pressure was used throughout (276 kPa). The $\mathrm{GC}$ oven was programed as so: $50{ }^{\circ} \mathrm{C}$ (isothermal for $2 \mathrm{~min}$ ), then $7{ }^{\circ} \mathrm{C} / \mathrm{min}$ until $240{ }^{\circ} \mathrm{C}$ and then $25^{\circ} \mathrm{C} / \mathrm{min}$ until $300{ }^{\circ} \mathrm{C}$ (isothermal for $3 \mathrm{~min}$ ). The second column was held at a temperature $20{ }^{\circ} \mathrm{C}$ higher than the first, however, the programed temperature changes remained the same. The transfer line was maintained at $260{ }^{\circ} \mathrm{C}$ and the source temperature at $200{ }^{\circ} \mathrm{C}$. The modulation time was $4 \mathrm{~s}$. The mass range 45 to $700 \mathrm{amu}$ with 100 spectra/s were measured using electron impact ionization $(70 \mathrm{eV})$.

All raw data processing was performed in ChromaTOF v4.32 (LECO Corporation, St. Joseph, MI). Peaks were selected automatically using the inbuilt spectral deconvolution using a peak width of $0.2 \mathrm{~s}$. Any peak with a signal to noise ratio $>100$ were rejected. For the majority of compounds the peak areas from the total ion chromatogram was used. However, if the metabolites were quantified directly in the ChromaTOF software then the peak areas of the selected $\mathrm{m} / \mathrm{z}$ were used.

The output text files from ChromaTOF were then imported into the in housed developed software Guineu (29). This software was used for aligning and normalizing the compounds for further analysis. Since the second column is so short its contribution to the RI was considered negligible so the RI could be directly compared to spectral libraries. The spectra were aligned in the first dimension using these RI values. To further align the spectra retention times and spectra were used as a second dimension. Following alignment, the following criteria was used to filter the data to ensure positive identification: (1) spectral 
similarity $>850$, (2) the maximum difference between RI values and literature values $<25$. Additionally each metabolite had to appear in $70 \%$ of the samples run in in each of the four original study groups. (21) This resulted in a total of 465 metabolites. All literature values were obtained from the NIST 2008 ass Spectral Library.

Further identification of unknown metabolites was performed using $\mathrm{GC} \times \mathrm{GC}$ equipped with high-resolution MS system and both electron impact and chemical ionization in conjunction with the GOLM database. (32) Metabolites that appeared in less than $70 \%$ of the samples were excluded from the subsequent analysis. In total 465 metabolites were detected using these criteria. Any metabolites that were identified as drugs such as propofol or ibuprofen were excluded from the data set as described previously. (21) Additionally, downstream metabolites of these compounds were excluded by examining which other metabolites highly correlated to the drug. This resulted in a total number of 455 metabolites, which were used in subsequent analysis.

The orders of both sample preparation and analysis were randomized, and a set of controls samples (pooled serum samples), standards and blank samples (solvent blank) was analyzed together with the samples. The day-to-day variation of internal standards added to all samples was on average $17.3 \%$ and $12.3 \%$ in the discovery and validation sets, respectively. The dayto-day variation in control serum samples $(n=31)$ of the quantified metabolites was $18.0 \%$ and $9.2 \%$ in the discovery and validation sets, respectively. The linear range of the method is from 0.1 to $120 \mathrm{ng} / \mu \mathrm{l}$ for quantified metabolites, with limit of quantification (LOQ, S/N 10) ranging from 0.005 to $0.1 \mathrm{ng} / \mu \mathrm{l}$. (33)

Imaging predictions - logistic regression modelling

In order to generate a classification model, 1000 cross-validated logistic regression models were generated from the Turku (discovery cohort) data. The models were cross-validated by 
selecting the best model from a group of 10 where $10 \%$ of the discovery data was withheld and used as a prediction set. The models were selected based on the area under the curve (AUC) of the resultant receiver operator curve (ROC). Once the 1000 models had been generated from the Turku data, the models were validated with the data from Cambridge. The need for a discovery and validation cohorts prevents the overfitting of the data as commonly seen in multivariate modelling of the data. To select the optimal combination of metabolites for the linear regression, we introduced metabolites in an iterative manner starting with all metabolites that had a $\mathrm{Q}$ value $<0.05$. To select the base model for the next iteration, the model with the highest independently validated (using the Cambridge data) AUC was selected. If the AUCs were the same for multiple metabolites, all these were selected for the next iteration.

\section{Protein analysis}

GFAP and UCH-L1 were measured using a Randox biochip (Randox Laboratories Ltd, Crumlin, Country Antrim) as described elsewhere. (20)

\section{Statistical Analysis}

The protein and metabolite data was processed as described previously. (21) The statistical analysis was performed in MATLAB 2016a (Mathworks Inc., Natick MA) using the PLS toolbox 8.1 (Eigenvector Research Inc., Manson, WA) or GraphPad Prism 7.03 (GraphPad Software Inc. La Jolla, CA).

For metabolite and protein values that were marked zero, we imputed a value, which was equal to half the minimum for that analyte across all the samples. In order to correct for the non-normally distributed data, all values were log transformed prior to further analysis. For each of the four aims in the study, a Student's t-test was performed to identify the metabolites, which differed significantly between the two groups. False Discovery Rates 
(FDR) were calculated to correct for multiple comparisons. (34) Q values less than 0.05 were considered significant. Differences in protein and metabolite concentrations were assessed with Welch's corrected t-test due to the differences in variances between the groups.

The regression curve was calculated by linear regression and the residual values were plotted on a separate figure. Metabolites were deemed to be outside the regression model if the residual value was greater than two standard deviations. 


\section{Results}

\section{Patient Inclusion}

We included all patients $(n=210)$ who had sustained TBI (regardless of severity) from both Turku, Finland $(n=144)$ and Cambridge, UK $(n=66)$. The flow chart for patient selection is shown in Figure 1. The serum metabolite levels measured from orthopedic controls from both Turku $(\mathrm{n}==28)$ and Cambridge $(\mathrm{n}=27)$ were used in the brain microdialysate analysis to normalize the metabolite concentrations. These patients had other peripheral injuries but with no evidence of head trauma. (21) For a flow chart of patient selection please see Figure 1

Serum metabolites can predict the need for a CT scan in a multi-center setting

Using a univariate approach (Welch t-test with FDR correction) we identified 36 metabolites that differed between the patients who had traumatic intracranial findings (CT positive) as compared to patients with negative CT findings (CT negative) (Table 2). Next, we built a logistic regression model to predict which patients with TBI would be CT positive. The iterative process resulted in a predictive model (sensitivity $=0.73$, specificity $=0.64$; Figure 2A), which utilized six metabolites (scatter plots shown in Figure 2B-H), including two amino acids, three sugar derivatives and one ketoacid. The model had good predictive accuracy in the discovery cohort, Turku (AUC $=0.77$; 95\% CI: 0.69-0.87) and the validation group, Cambridge $(\mathrm{AUC}=0.73 ; 95 \%$ CI: 0.73-0.74). Next, we hypothesized that a combination of metabolites and proteins might furnish a more accurate prediction model. When the protein data was included in the logistic regression model, the predictive accuracy did not improve to a meaningful extent: Turku, discovery cohort, AUC $=0.78$ (95\% CI: 0.690.87), Cambridge, validation cohort, $\mathrm{AUC}=0.73$ (95\% CI: 0.73-0.74). 
GFAP and UCH-L1 validate poorly in a multi-center setting when predicting the need for a CT scan

We measured GFAP and UCH-L1 in two independent cohorts acquired from Turku (discovery cohort, Finland) and Cambridge (validation cohort, UK). There were six samples from Turku and three from Cambridge, which did not have protein data available. Using a univariate approach (Welch's t-test) there were clear increases in both GFAP ( $p_{\text {turku }}=0.0002$; $\left.p_{\text {cambridge }}=0.0004\right)$ and UCH-L1 $\left(p_{\text {turku }}=0.0094\right)$ in patients who had abnormalities on the CT scan, when compared to TBI patients with no CT pathology (Figure 3 A-D). The one exception to this were the UCH-L1 levels in the Cambridge cohort which did not differ between the groups $\left(p_{\text {cambridge }}=0.1823\right)$. Using the logistic regression modelling in the Turku cohort, it was possible to generate models using GFAP (AUC $=0.73 ; 95 \%$ CI: 0.64-0.83), $\mathrm{UCH}-\mathrm{L} 1(\mathrm{AUC}=0.71 ; 95 \% \mathrm{CI}: 0.62-0.85)$ or a combination of both (Figure $3 \mathrm{E}$ AUC $=0.73$; 95\% CI: 0.62-0.86), that could predict abnormalities on a CT scan (Marshall Grade $>1$ ). When the Cambridge data was used for validation, the models did not perform as expected, with GFAP (Figure 3E AUC $=0.64 ; 95 \%$ CI: 0.64-0.64), UCH-L1 $(\mathrm{AUC}=0.58 ; 95 \% \mathrm{CI}$ : $0.58-0.58)$ and the combination model $(\mathrm{AUC}=0.64 ; 95 \% \mathrm{CI}: 0.63-0.64)$ all giving poorer predictive accuracy.

Serum metabolites can be used to predict other CT based features

When comparing diffuse lesions (Marshall Grade = 2-4) with mass lesions (Marshall Grade = 5-6), we identified six metabolites (Table 3).

Given that only a limited number of metabolites changed significantly between the groups for aim 2, we built linear regression models for these. When classifying the CT positive TBI patients into those who had diffuse injuries compared to mass lesions (Aim 2), the resulting 
model performed well with the Turku data AUC $=0.87$ (95\% CI: 0.75-0.94) and crossvalidated to some degree in the Cambridge data $\mathrm{AUC}=0.68$ (95\% CI: 0.68-0.69) (Figure 4A). The logistic regression model comprised three metabolites (Figure 4B-D), an unknown phenolic compound, isovalerylglucuronide and 2-hydroxybutyric acid. When comparing surgically operated mass lesions to non-surgically operated mass lesions, the model had good predictive accuracy in the Turku dataset AUC $=0.84$ (95\% CI: 0.70-0.90). However, this model did not validate as well when applied to the Cambridge dataset $\mathrm{AUC}=0.60(95 \% \mathrm{CI}$ : 0.60-0.61). This logistic regression model comprised two metabolites, a sugar derivative and octanoic acid.

Metabolites measured in the serum associate with levels measured in brain microdialysates (BMD)

In our previous study the levels of metabolites were determined in the brain microdialysate (n =12) (21). The results from this analysis are reproduced here (Figure 5A). This original study compared the metabolite levels to orthopedic controls. (21) Since the metabolites used in the linear prediction models differed from those that associated with the severity of TBI, a single regression line was calculated from the data as opposed to the two generated in the previous study. (21) In order to understand the origins of the metabolite changes in the present study, we calculated the vertical distance (residual, e) between the predicted value and the measured value for each metabolite (Figure 5B). The sugar derivatives inositol $(\mathrm{e}=0.018)$, ribonic acid $(\mathrm{e}=0.079)$, pentitol 3-desoxy $(\mathrm{e}=-0.316)$, and isovalerylglucuronide $(\mathrm{e}=0.355)$ had low residual values, demonstrating that the metabolite concentrations are closely associated between BMD and serum. However, the unknown phenolic compound $(\mathrm{e}=3.49)$ did not fit with the regression line. Additionally, there were two metabolites, acetoacetic acid and an unknown amino acid, that were not detected in the BMD at all. 


\section{Discussion}

There is an unmet clinical need to distinguish if a patient requires a head CT scan following TBI. Here we demonstrate the effectiveness of circulating metabolites in stratification of patients based on their $\mathrm{CT}$ imaging. Our results show that metabolites can generate better cross-validated predictions when compared to protein measures. We have identified a metabolite panel, which can discriminate between diffuse brain injury and mass lesions as observed in the CT scan.

One of the key strengths of this study is the use of two independent cohorts, which enables us to cross-validate the predictive models in a multi-center setting. We were surprised by the poor performance of the protein measurements in a multicenter setting compared to the metabolomics results, especially given the previously published data which showed some promise. (1, 4-6) The primary limitation of the earlier studies was the lack of external validation and potentially the wide variety of analytical platforms used. The method in this study was a novel chip based assay. Our results suggest that even though the GFAP and UCH-L1 concentrations increase following the injury, they do not do so in a consistent enough for logistic regression modelling. There are many possible explanations why metabolites in the blood can be more suitable to model the TBI-related changes in the brain as proteins. One of the key factors is the relative size difference between metabolites (100$1500 \mathrm{Da})$ and proteins $(10,000-100,000 \mathrm{Da})$. Metabolites can cross the BBB more readily following the injury for several reasons, ranging from BBB breakdown (35) to subtle changes in astrocytes in response to inflammatory stimuli (36) or alterations in the hypoxia-inducible factor-1 $\alpha$ pathway and elevated expression of aquaporin-4. (37) The changes in the proteins, especially GFAP, could arise from changes to the BBB itself as opposed to changes within the brain parenchyma. It could be that the protein measures are more sensitive to differences in sample handling. (38-40) This limitation could in principle be solved by imposing stricter 
standard operating procedures (SOPs) in the clinic. The robustness of a predictive model to sample handling is a key feature when developing a clinically relevant tool, (39) as the wider the implementation, the harder it is to achieve compliance with complicated SOPs. Aside from proteins there is a set of clinical guidelines utilized in Europe assessing the need for a CT scan based on clinical data. Clinical CT prediction tools, such as the UK NICE Criteria, can provide useful bases for assessing the need for a CT scan following TBI. (41) However, such schemes are imperfect, and could be improved by a simple blood test, which could be developed with further work on a metabolomics assay. However, a larger study directly comparing the NICE guidelines to a metabolomics assay would need to be performed to fully assess the clinical utility of a metabolomics based assay.

Given that we are determining the serum metabolites following a brain injury, it is challenging to identify the sources of the variation observed. All the sugar-related metabolites, pentitol 3-desoxy, inositol, isovalerylglucuronide, and ribonic acid, which generated predictive models fitted the correlation model. All these sugar derivatives increased in concentration when there was detectable pathology on a CT scan or a presence of a mass lesion suggesting that the source of these changes originate from the brain. There have been several studies that demonstrate altered glucose metabolism $(22,42)$ following TBI, which could also explain the increase of sugar derivatives in the circulation.

2-aminobutyric acid is another metabolite fitting the correlation model. Its concentration was lower in patients with detectable CT features. These reduced levels in the serum could indicate increased oxidative stress (43) in the brain. Elevated oxidative stress markers have been observed in the CSF of patients with severe TBI (44) reinforcing the probability that changes in 2-aminobutyric acid originated within the CNS. Some metabolites, did not fit the correlation model (an unknown phenolic compound) or were not detected in the BMD 
(acetoacetic acid and an unknown amino acid). While determining the identity and/or source of these metabolites requires further work, we have some pointers in this area.

We speculate that the unknown phenolic compound may be propofol, or one of its metabolites. Propofol is an intravenous anaesthetic which is commonly used for sedation in patients with moderate to severe TBI who require tracheal intubation and mechanical ventilation for airway protection and control of ventilation. (45) It is also important to recognize that propofol, which is highly lipophilic, is solubilized in an oil in water emulsion which contains $100 \mathrm{mg}$ soya bean oil per milliliter. (46) The additional lipid load provided by this formulation would only have been present in patients with more severe TBI, and hence a greater likelihood of having a positive CT, and consequently represents a potential confound. Other potential explanations also need to be considered. While starvation ketosis cannot be excluded, this seems an unlikely cause after less than a day's starvation. Further, the change in systemic levels potentially suggests a source other than the brain, of metabolite changes following TBI. This is not unexpected, as clear evidence exists that brain injuries result in changes in the periphery. Immediately after the injury there is an induction of acute phase proteins in the liver. $(47,48)$ Additionally, there are modulation of proteins, such as peroxisome proliferator-activated receptor alpha, (49) which play a key role in lipid homeostasis. One caveat about the metabolites absent in the BMD is that the metabolite concentration compared to serum is very low. However, highly lipophilic metabolites may not partition into the aqueous microdialysis fluid, and hence may not have been detected by BMD.

The two metabolites, not observed in the BMD, could be there but at low levels. They still not fit the correlation model, thus indicating a non-brain derived origin to the change in concentration of these metabolites. From these three metabolites only acetoacetic acid can be 
identified. It is a ketone body produced in the liver. (50) These act as an energy source following injury. (51) Fasting to induce ketosis has been shown to have a neuroprotective effect following TBI. (52) The other non-identified metabolites make it hard to interpret their biological function. It would appear that changes in these metabolites are not brain derived.

There are several limitations to this current study. First, though the recruitment process with identical inclusion and exclusion criteria of TBI care consortium was carefully planned while preparing the study protocol, the representativeness of included population varies between the Turku and Cambridge cohorts. This may partially be due to fact that varying recruitment resources and information transfer about the inbound patients at the emergency departments. In Cambridge, only $17 \%$ of the patients with suspected TBI were included in the study, while in Turku, $33 \%$ were included. Both of the institutions are university hospitals with a distinction that Turku University Hospital serves as a both primary emergency center and referral center whereas Addenbrooke's Hospital serves as a tertiary referral center. This might have an effect on the characteristics of trauma patients admitted.

Second, the mechanism of injury and the outcome as measured by the extended Glasgow Outcome Scale were different in the two cohorts (21) and the patients were older and had sustained more severe injuries based on the imaging findings especially in mTBI group in Turku cohort. The difference between two cohorts is presumably the reason why GFAP and UCH-L1 validated poorly in Cambridge cohort highlighting the problems of these protein biomarkers in acute diagnostics in cases of milder TBIs.

In conclusion, it is likely that there is a notable variation in the representativeness, preexisting health problems based on the differences in cohort demographics and injury characteristics between the study cohorts. Hence the Turku cohort, is older and more seriously injured. To fully explore these patients, a larger study is necessary. 
The metabolites identified from the full spectrum generated a model that internally crossvalidated in the Turku cohort, which suggests that metabolites will be the best source of providing $\mathrm{CT}$ predictive models in the future

The mass spectrometry technology used in this study is a profiling research method, as opposed to a targeted assay, which provides broad analytical coverage and high sensitivity but is not fully quantitative and thus not suitable for applications in the healthcare setting. (53) The metabolites identified could be quantified using a targeted MS approach for selected metabolites. Such targeted assays and required infrastructure are readily available in typical clinical chemistry laboratories. The manual sample processing step which is required for the GC based profiling method can be simplified by either automating it or switching to a quantitative LC based method. The cost of a targeted MS assay is far less than a CT scan when considering interpretation cost. Such assays would reduce the cost of care following TBI and ensure patients only receive the X-ray radiation from a CT scan when required. Additionally, the fact that this was a global metabolite screen could contribute to the lower sensitivity and specificity observed in the metabolite model. A targeted screen focused on the metabolites identified in this manuscript should help increase this sensitivity and specificity. However, it is worth noting that the metabolites did outperform the proteins when employed in a multi-center setting which is more clinically relevant.

\section{Conclusion}

This study is the first to demonstrate the use of metabolomics in the serum for classifying patients with TBI who need a CT scan. We have demonstrated that the protein serum markers GFAP and UCH-L1 perform poorly in a multi-centre setting. Additionally, we have identified metabolites, which can predict whether the patient has diffuse injury or mass lesion. The 
metabolite models could have an impact on the ability to provide personalized healthcare to patients with TBI and prevent unnecessary CT scans.

\section{Acknowledgements}

The work was supported by the EU FP7 project TBIcare (Project ref. 270259 to MO DM and OT), by the GE-NFL Head Health Challenge I Award (grant no. 7620 to MO and TH), by Goverment's Special Financial Transfer tied to academic research in Health Sciences (Finland) (to JPP and RSKT.), and by personal grant from Emil Aaltonen Foundation and Finnish Brain Foundation (to JPP). Support for P.J.H. - National Institute for Health Research (NIHR) Research Professorship; NIHR Biomedical Research Centre (NIHR BRC) Cambridge. Support for K.L.H.C. - NIHR BRC Cambridge. This work was supported by the NIHR Biomedical Research Centre in Cambridge. VFJN is supported by an Academy of Medical Sciences / The Health Foundation Fellowship.

We would like to thank all the study participants of TBI care for providing the samples used in this study. We would also like to thank Mark Van Gils who coordinated the TBI care project. Furthermore, we would like to thank Anna-Liisa Ruuskepää for technical assistance in metabolomics analysis. The authors would also like to thank research nurses Patricia Bertenyi, and Satu Timlin for their valuable contribution to this study.

\section{Author Contribution}

The study was designed by JPP, AMD, OT, TH, and MO. The study protocol was designed by TH and MO. Patients were recruited, clinical data collected, and blood samples by JPP, RSKT, AJK, HAS, AK, H-RM, JT, JPC, and JF. KLHC and PJH performed brain microdialysis studies. IM and TH acquired and processed the metabolomics analysis. TH identified the unknown metabolites identified by the modelling. AMD performed all the univariate and linear regression modelling. AMD and MO drafted the first versions of the 
manuscript. JPP, DM, OT and TH provided critical contributions. All authors reviewed edited and approved the final version of the manuscript.

\section{Conflicts of Interest}

AMD, JPP, TH, OT, and MO are all co-applicants on a patent relating to the use of metabolomics for the diagnosis and prognosis of mTBI. 


\section{References}

1. Posti, J.P., Takala, R.S., Runtti, H., Newcombe, V.F., Outtrim, J., Katila, A.J., Frantzén, J., Ala-Seppälä, H., Coles, J.P. and Hossain, M.I. (2016). The levels of glial fibrillary acidic protein and ubiquitin $\mathrm{C}$-terminal hydrolase-L1 during the first week after a traumatic brain injury: correlations with clinical and imaging findings. Neurosurgery 79, 456-464.

2. Haydel , M.J., Preston, C.A., Mills , T.J., Luber, S., Blaudeau , E. and DeBlieux , P.M.C. (2000). Indications for Computed Tomography in Patients with Minor Head Injury. New England Journal of Medicine 343, 100-105.

3. Biberthaler, P., Linsenmeier, U., Pfeifer, K.-J., Kroetz, M., Mussack, T., Kanz, K.-G., Hoecherl, E.F., Jonas, F., Marzi, I. and Leucht, P. (2006). Serum S-100B concentration provides additional information fot the indication of computed tomography in patients after minor head injury: a prospective multicenter study. Shock 25, 446-453.

4. Pelinka, L.E., Kroepfl, A., Leixnering, M., Buchinger, W., Raabe, A. and Redl, H. (2004). GFAP versus S100B in serum after traumatic brain injury: relationship to brain damage and outcome. J. Neurotrauma 21, 1553-1561.

5. Papa, L., Lewis, L.M., Silvestri, S., Falk, J.L., Giordano, P., Brophy, G.M., Demery, J.A., Liu, M.C., Mo, J. and Akinyi, L. (2012). Serum levels of ubiquitin C-terminal hydrolase distinguish mild traumatic brain injury from trauma controls and are elevated in mild and moderate traumatic brain injury patients with intracranial lesions and neurosurgical intervention. Journal of Trauma and Acute Care Surgery 72, 1335-1344.

6. Papa, L., Silvestri, S., Brophy, G., Giordano, P., Falk, J., Braga, C., Tan, C., Ameli, N., Demery, J. and Dixit, N. (2014). GFAP out-performs S100B in detecting traumatic intracranial lesions on $\mathrm{CT}$ in trauma patients with mild traumatic brain injury and those with extracranial lesions. J. Neurotrauma 31, 1815-1822. 
7. Anderson, V.A., Catroppa, C., Haritou, F., Morse, S., Pentland, L., Rosenfeld, J. and Stargatt, R. (2001). Predictors of acute child and family outcome following traumatic brain injury in children. Pediatr. Neurosurg. 34, 138-148.

8. Torabian, S. and Kashani-Sabet, M. (2005). Biomarkers for melanoma. Curr. Opin. Oncol. $17,167-171$.

9. Koh, S.X. and Lee, J.K. (2014). S100B as a marker for brain damage and blood-brain barrier disruption following exercise. Sports Med 44, 369-385.

10. Pelinka, L.E., Toegel, E., Mauritz, W. and Redl, H. (2003). Serum S 100 B: a marker of brain damage in traumatic brain injury with and without multiple trauma. Shock 19, 195-200. 11. Thelin, E.P., Jeppsson, E., Frostell, A., Svensson, M., Mondello, S., Bellander, B.-M. and Nelson, D.W. (2016). Utility of neuron-specific enolase in traumatic brain injury; relations to S100B levels, outcome, and extracranial injury severity. Critical Care 20, 285.

12. Sofroniew, M.V. and Vinters, H.V. (2010). Astrocytes: biology and pathology. Acta neuropathologica $119,7-35$.

13. Bleeker, S., Moll, H., Steyerberg, E., Donders, A., Derksen-Lubsen, G., Grobbee, D. and Moons, K. (2003). External validation is necessary in prediction research:: A clinical example. Journal of clinical epidemiology 56, 826-832.

14. Papa, L., Akinyi, L., Liu, M.C., Pineda, J.A., Tepas III, J.J., Oli, M.W., Zheng, W., Robinson, G., Robicsek, S.A. and Gabrielli, A. (2010). Ubiquitin C-terminal hydrolase is a novel biomarker in humans for severe traumatic brain injury. Critical care medicine 38, 138.

15. Takala, R.S., Posti, J.P., Runtti, H., Newcombe, V.F., Outtrim, J., Katila, A.J., Frantzén, J., Ala-Seppälä, H., Kyllönen, A. and Maanpää, H.-R. (2016). Glial fibrillary acidic protein and ubiquitin C-terminal hydrolase-L1 as outcome predictors in traumatic brain injury. World neurosurgery $87,8-20$. 
16. Prakash, R. and Carmichael, S.T. (2015). Blood- brain barrier breakdown and neovascularization processes after stroke and traumatic brain injury. Current opinion in neurology $28,556-564$.

17. Price, L., Wilson, C. and Grant, G. (2016). Blood-brain barrier pathophysiology following traumatic brain injury.

18. Garrison, C., Dougherty, P., Kajander, K. and Carlton, S. (1991). Staining of glial fibrillary acidic protein (GFAP) in lumbar spinal cord increases following a sciatic nerve constriction injury. Brain Res. 565, 1-7.

19. Mancardi, G., Cadoni, A., Tabaton, M., Schenone, A., Zicca, A., De Martini, I., Bianchini, D., Damiani, G. and Zaccheo, D. (1991). Schwann cell GFAP expression increases in axonal neuropathies. Journal of the neurological sciences 102, 177-183.

20. Posti, J.P., Hossain, I., Takala, R.S., Liedes, H., Newcombe, V., Outtrim, J., Katila, A.J., Frantzen, J., Ala-Seppala, H., Coles, J.P., Kyllonen, A., Maanpaa, H.R., Tallus, J., Hutchinson, P.J., van Gils, M., Menon, D.K. and Tenovuo, O. (2017). Glial Fibrillary Acidic Protein and Ubiquitin C-Terminal Hydrolase-L1 Are Not Specific Biomarkers for Mild CTNegative Traumatic Brain Injury. J. Neurotrauma.

21. Orešič, M., Posti, J.P., Kamstrup-Nielsen, M.H., Takala, R.S., Lingsma, H.F., Mattila, I., Jäntti, S., Katila, A.J., Carpenter, K.L., Ala-Seppälä, H., Kyllönen, A., Maanpää, H.R., Tallus, J., Coles, J.P., Heino, I., Frantzén, J., Hutchinson, P.J., Menon, D.K., Tenovuo, O. and Hyötyläinen, T. (2016). Human Serum Metabolites Associate With Severity and Patient Outcomes in Traumatic Brain Injury. EBioMedicine.

22. Glenn, T.C., Kelly, D.F., Boscardin, W.J., McArthur, D.L., Vespa, P., Oertel, M., Hovda, D.A., Bergsneider, M., Hillered, L. and Martin, N.A. (2003). Energy dysfunction as a predictor of outcome after moderate or severe head injury: indices of oxygen, glucose, and lactate metabolism. Journal of Cerebral Blood Flow \& Metabolism 23, 1239-1250. 
23. Wolahan, S.M., Hirt, D., Braas, D. and Glenn, T.C. (2016). Role of Metabolomics in Traumatic Brain Injury Research. Neurosurgery Clinics of North America 27, 465-472.

24. Dickens, A.M., Larkin, J.R., Griffin, J.L., Cavey, A., Matthews, L., Turner, M.R., Wilcock, G.K., Davis, B.G., Claridge, T.D.W., Palace, J., Anthony, D.C. and Sibson, N.R. (2014). A type 2 biomarker separates relapsing-remitting from secondary progressive multiple sclerosis. Neurology 83, 1492-1499.

25. Antila, K., Lötjönen, J., Thurfjell, L., Laine, J., Massimini, M., Rueckert, D., Zubarev, R.A., Orešič, M., van Gils, M. and Mattila, J. (2013). The PredictAD project: development of novel biomarkers and analysis software for early diagnosis of the Alzheimer's disease. Interface Focus 3, 20120072.

26. Suvitaival, T., Mantere, O., Kieseppä, T., Mattila, I., Pöhö, P., Hyötyläinen, T., Suvisaari, J. and Orešič, M. (2016). Serum metabolite profile associates with the development of metabolic co-morbidities in first-episode psychosis. Translational Psychiatry 6, e951.

27. Bahado-Singh, R.O., Graham, S.F., Han, B., Turkoglu, O., Ziadeh, J., Mandal, R., Er, A., Wishart, D.S. and Stahel, P.L. (2016). Serum metabolomic markers for traumatic brain injury: a mouse model. Metabolomics 12, 100.

28. Posti, J.P., Dickens, A.M., Orešič, M., Hyötyläinen, T. and Tenovuo, O. (2017). Metabolomics profiling as a diagnostic tool in severe traumatic brain injury. Frontiers in neurology 8,398 .

29. Castillo, S., Mattila, I., Miettinen, J., Orešič, M. and Hyötyläinen, T. (2011). Data analysis tool for comprehensive two-dimensional gas chromatography/time-of-flight mass spectrometry. Analytical chemistry 83, 3058-3067.

30. Bullock, M.R. and Povlishock, J.T. (2007). Guidelines for the management of severe traumatic brain injury. Editor's Commentary. J. Neurotrauma 24, 2 p preceding S1. 
31. Marshall, L., Marshall, S.B., Klauber, M., Van Berkum, C.M., Eisenberg, H., Jane, J., Luerssen, T., Marmarou, A. and Foulkes, M. (1992). The diagnosis of head injury requires a classification based on computed axial tomography. J. Neurotrauma 9, S287.

32. Kopka, J., Schauer, N., Krueger, S., Birkemeyer, C., Usadel, B., Bergmüller, E., Dörmann, P., Weckwerth, W., Gibon, Y. and Stitt, M. (2005). GMD@ CSB. DB: the Golm metabolome database. Bioinformatics 21, 1635-1638.

33. Hartonen, M., Mattila, I., Ruskeepää, A.-L., Orešič, M. and Hyötyläinen, T. (2013). Characterization of cerebrospinal fluid by comprehensive two-dimensional gas chromatography coupled to time-of-flight mass spectrometry. J. Chromatogr. A 1293, 142149.

34. Storey, J.D. (2002). A direct approach to false discovery rates. Journal of the Royal Statistical Society: Series B (Statistical Methodology) 64, 479-498.

35. Shlosberg, D., Benifla, M., Kaufer, D. and Friedman, A. (2010). Blood-brain barrier breakdown as a therapeutic target in traumatic brain injury. Nature Reviews Neurology 6, 393-403.

36. Chen, Y. and Swanson, R.A. (2003). Astrocytes and brain injury. Journal of Cerebral Blood Flow \& Metabolism 23, 137-149.

37. Higashida, T., Kreipke, C.W., Rafols, J.A., Peng, C., Schafer, S., Schafer, P., Ding, J.Y., Dornbos III, D., Li, X. and Guthikonda, M. (2011). The role of hypoxia-inducible factor-1 $\alpha$, aquaporin-4, and matrix metalloproteinase-9 in blood-brain barrier disruption and brain edema after traumatic brain injury: laboratory investigation. Journal of neurosurgery 114, 92101.

38. Mitchell, B.L., Yasui, Y., Li, C.I., Fitzpatrick, A.L. and Lampe, P.D. (2005). Impact of freeze-thaw cycles and storage time on plasma samples used in mass spectrometry based biomarker discovery projects. Cancer informatics 1. 
39. Jørgenrud, B., Jäntti, S., Mattila, I., Pöhö, P., Rønningen, K.S., Yki-Järvinen, H., Orešič, M. and Hyötyläinen, T. (2015). The influence of sample collection methodology and sample preprocessing on the blood metabolic profile. Bioanalysis 7, 991-1006.

40. Hsieh, S.Y., Chen, R.K., Pan, Y.H. and Lee, H.L. (2006). Systematical evaluation of the effects of sample collection procedures on low-molecular-weight serum/plasma proteome profiling. Proteomics 6, 3189-3198.

41. Hodgkinson, S., Pollit, V., Sharpin, C. and Lecky, F. (2014). Early management of head injury: summary of updated NICE guidance. BMJ: British Medical Journal (Online) 348.

42. Clausen, F., Hillered, L. and Gustafsson, J. (2011). Cerebral glucose metabolism after traumatic brain injury in the rat studied by $13 \mathrm{C}$-glucose and microdialysis. Acta Neurochir. $153,653-658$.

43. Soga, T., Baran, R., Suematsu, M., Ueno, Y., Ikeda, S., Sakurakawa, T., Kakazu, Y., Ishikawa, T., Robert, M. and Nishioka, T. (2006). Differential metabolomics reveals ophthalmic acid as an oxidative stress biomarker indicating hepatic glutathione consumption. Journal of Biological Chemistry 281, 16768-16776.

44. Bayir, H., Kagan, V.E., Tyurina, Y.Y., Tyurin, V., Ruppel, R.A., Adelson, P.D., Graham, S.H., Janesko, K., Clark, R.S. and Kochanek, P.M. (2002). Assessment of antioxidant reserves and oxidative stress in cerebrospinal fluid after severe traumatic brain injury in infants and children. Pediatr. Res. 51, 571-578.

45. Oddo, M., Crippa, I.A., Mehta, S., Menon, D., Payen, J.-F., Taccone, F.S. and Citerio, G. (2016). Optimizing sedation in patients with acute brain injury. Critical Care 20, 128.

46.

FDA

(2008).

https://www.accessdata.fda.gov/drugsatfda_docs/label/2008/019627s0461bl.pdf.

47. Campbell, S.J., Hughes, P.M., Iredale, J.P., Wilcockson, D.C., Waters, S., Docagne, F., Perry, V.H. and Anthony, D.C. (2003). CINC-1 is identified as an acute-phase protein 
induced by focal brain injury causing leukocyte mobilization and liver injury. The FASEB Journal.

48. Wilcockson, D.C., Campbell, S.J., Anthony, D.C. and Perry, V.H. (2002). The systemic and local acute phase response following acute brain injury. Journal of Cerebral Blood Flow \& Metabolism 22, 318-326.

49. Dickens, A.M., Tovar, Y.R.L.B., Yoo, S.W., Trout, A.L., Bae, M., Kanmogne, M., Megra, B., Williams, D.W., Witwer, K.W., Gacias, M., Tabatadze, N., Cole, R.N., Casaccia, P., Berman, J.W., Anthony, D.C. and Haughey, N.J. (2017). Astrocyte-shed extracellular vesicles regulate the peripheral leukocyte response to inflammatory brain lesions. Science signaling 10 .

50. Newman, J.C. and Verdin, E. (2014). Ketone bodies as signaling metabolites. Trends Endocrinol. Metab. 25, 42-52.

51. Prins, M.L. (2008). Cerebral metabolic adaptation and ketone metabolism after brain injury. Journal of Cerebral Blood Flow \& Metabolism 28, 1-16.

52. Davis, L.M., Pauly, J.R., Readnower, R.D., Rho, J.M. and Sullivan, P.G. (2008). Fasting is neuroprotective following traumatic brain injury. J. Neurosci. Res. 86, 1812-1822.

53. Tebani, A., Abily-Donval, L., Afonso, C., Marret, S. and Bekri, S. (2016). Clinical metabolomics: The new metabolic window for inborn errors of metabolism investigations in the post-genomic era. International Journal of Molecular Sciences 17, 1167. 
Serum metabolites predict the need for associate with CT seanfindings following TBI

Running head: Serum metabolomics predictsassociates with CT imaging in TBI

Authors: Alex M. Dickens, D.Phil. ${ }^{1 *}$, Jussi P. Posti, MD, PhD ${ }^{2,3,4 *}$, Riikka S.K. Takala, $\mathrm{MD}, \mathrm{PhD}^{5}$, Henna Ala-Seppälä, $\mathrm{MD}^{3}$, Ismo Mattila ${ }^{6}$, MSc, Jonathan P. Coles, MD, $\mathrm{PhD}^{7}$, Janek Frantzén, MD, $\mathrm{PhD}^{2,3,4}$, Peter J. Hutchinson, FRCS (SN), $\mathrm{PhD}^{8}$, Ari J. Katila, $\mathrm{MD}^{5}$, Anna Kyllönen, $\mathrm{MD}^{3}$, Henna-Riikka Maanpää, $\mathrm{MD}^{3}$, Virginia Newcombe, $\mathrm{MD}, \mathrm{PhD}^{7}$, Joanne Outtrim, $\mathrm{RN}^{7}$, Jussi Tallus, $\mathrm{MD}^{3}$, Keri L H Carpenter, $\mathrm{PhD}^{8}$, David K. Menon, MD, $\mathrm{PhD}^{7}$, Tuulia Hyötyläinen, $\mathrm{PhD}^{9}$, Olli Tenovuo, $\mathrm{MD}, \mathrm{PhD}^{2,3}$, and Matej Orešič, $\mathrm{PhD}^{1,10}$.

*These authors contributed equally to this work.

Affiliations: ${ }^{1}$ Turku Centre for Biotechnology, University of Turku, Finland; ${ }^{2}$ Turku Brain Injury Centre, Turku University Hospital, Finland; ${ }^{3}$ Department of Neurology, University of Turku, Finland; ${ }^{4}$ Division of Clinical Neurosciences, Department of Neurosurgery, Turku University Hospital, Finland; ${ }^{5}$ Perioperative Services, Intensive Care Medicine and Pain Management, Turku University Hospital and University of Turku, Finland, ${ }^{6}$ Steno Diabetes Center Copenhagen, DK-2820 Gentofte, Denmark. ${ }^{7}$ Division of Anaesthesia, Department of Medicine, University of Cambridge, Addenbrooke's Hospital, Cambridge, United Kingdom; ${ }^{8}$ Division of Neurosurgery, Department of Clinical Neurosciences, University of Cambridge, Addenbrooke's Hospital, Cambridge, United Kingdom, ${ }^{9}$ Department of Chemistry, Örebro University, Örebro, Sweden, and ${ }^{10}$ Schools of Medical Science, Örebro University, Örebro, Sweden.

Co-first and Corresponding Author: Alex M. Dickens

Turku Centre for Biotechnology

Tykistökatu 6

20520 Turku

FINLAND

Email: alex.dickens@utu.fi, Phone: +358294503798 Fax: +358 294505040

Mailing Addresses of all other authors:

Jussi P Posti

Division of Clinical Neurosciences, Department of Neurosurgery

Turku University Hospital

20521Turku

Finland

Email: Jussi.Posti@utu.fi, Phone: +358 2333 8585, Fax: +358 23133052

Riikka S.K. Takala 
Perioperative Services, Intensive Care Medicine and Pain

PO Box 52,

29521 Turku

Finland

Email: riikka.takala@gmail.com, Phone: +358 23338585 Fax: +358 23133960

Henna Ala-Seppälä

Department of Neurology

University of Turku

20014 Turku

Finland

Email: hmalse@utu.fi, Phone: +358 2333 8585, Fax: +358 294505040

Ismo Mattila

Steno Diabetes Center Copenhagen

Systems Medicine

Niels Steensensvej 2

DK-2820 Gentofte

Email: ismo.matias.mattila@regionh.dk, Phone: +45 3091 33 43, Fax: +358 294505040

Jonathan P. Coles

Division of Anaesthesia

Department of Medicine

University of Cambridge

Addenbrooke's Hospital

Cambridge

United Kingdom

CB2 2QQ

Email: jpc44@wbic.cam.ac.uk, Phone:+44 1223 336946, Fax:+44 1223217887

Janek Frantzén

Division of Clinical Neurosciences, Department of Neurosurgery

Turku University Hospital

20521

Turku

Finland

Email: Janek.frantzen@tyks.fi, Phone: +358 2 3130617, Fax: +358 23133052

Peter J. Hutchinson

Division of Neurosurgery

Department of Clinical Neurosciences

University of Cambridge

Box 167, Cambridge Biomedical Campus

Cambridge

CB2 0QQ

UK

Email: pjah2@cam.ac.uk, Phone: +44 1223 336946, Fax: +44 1223216926

Ari J. Katila 
Perioperative Services, Intensive Care Medicine and Pain

PO Box 52,

29521 Turku

Finland

Email: ari.katila@tyks.fi, Phone: +3582 3338585 Fax: +358 23133960

Anna Kyllönen

Department of Neurology

University of Turku

20014 Turku

Finland

Email: akkyll@utu.fi, Phone: +358 2333 8585, Fax: +358 294505040

Henna-Riikka Maanpää

Department of Neurology

University of Turku

20014 Turku

Finland

Email: hrmaan@utu.fi, Phone: +3582 333 8585, Fax: +358 294505040

Virginia Newcombe

Division of Anaesthesia

Department of Medicine

University of Cambridge

Addenbrooke's Hospital

Cambridge

United Kingdom

CB2 2QQ

Email: vfjn2@cam.ac.uk, Phone: +44 1223 336946, Fax: +44 1223217887

Joanne Outtrim

Division of Anaesthesia

Department of Medicine

University of Cambridge

Addenbrooke's Hospital

Cambridge

United Kingdom

CB2 2QQ

Email: jgo22@cam.ac.uk, Phone: +44 1223 336946, Fax: +44 1223217887

Jussi Tallus

Department of Neurology

University of Turku

20014 Turku

Finland

Email: jptall@utu.fi, Phone: +358 2333 8585, Fax: +358 294505040

\section{Keri L H Carpenter}

Division of Neurosurgery

Department of Clinical Neurosciences 
University of Cambridge

Box 167, Cambridge Biomedical Campus

Cambridge

CB2 0QQ

UK

Email: klc1000@wbic.cam.ac.uk, Phone: +44 1223 746453, Fax: +44 1223216926

David K. Menon

Division of Anaesthesia

Department of Medicine

University of Cambridge

Addenbrooke's Hospital

Cambridge

United Kingdom

CB2 2QQ

Email:dkm13@wbic.cam.ac.uk, Phone: +44 1223 217891, Fax: +44 1223217887

Tuulia Hyötyläinen

Department of Chemistry

University of Örebro

70285 Örebro

Sweden

Email: Tuulia.Hyotylainen@oru.se Phone: +46 19 303487, Fax: +358 294505040

Olli Tenovuo

Turku University Hospital

Kiinamyllynkatu 4-8

20520 Turku, Finland

Email: ollten@utu.fi, Phone: +358 50 4383802, Fax: +358 23132737

Matej Orešič

Turku Centre for Biotechnology

Tykistökatu 6

FI-20520 Turku

FINLAND

Email: matej.oresic@utu.fi Phone: +358 44972 6094, Fax: +358 294505040 
Abstract

There is a need to rapidly detect patients with traumatic brain injury (TBI) who require head hypothesized that serum metabolomics would be a useful tool for developing a set of biomarkers to determine the need for CT and to distinguish between different types of injuries observed.

-Logistic regression models using metabolite data from the discovery cohort ( $\mathrm{n}=144$, Turku, Finland) were used to distinguish between patients with traumatic intracranial findings and negative findings on head CT. The resultant models were then tested in the validation cohort ( $n=66$, Cambridge, UK). The levels of glial fibrillary acidic protein and ubiquitin C-terminal hydrolase-L1 were also quantified in the serum from the same patients.

-Despite there being significant differences in the protein biomarkers in patients with TBI, the model that determined the need for a CT scan validated poorly (AUC=0.64: Cambridge patients). However, using a combination of six metabolites (two amino acids, three sugar derivatives and one ketoacid) it was possible to discriminate patients with intracranial abnormalities on CT and patients with a normal CT (AUC $=0.77$ in Turku patients and AUC $=0.73$ in Cambridge patients). Furthermore, a combination of three metabolites could distinguish between diffuse brain injuries and mass lesions (AUC $=0.87$ in Turku patients and $\mathrm{AUC}=0.68$ in Cambridge patients).

This study identifies a set of validated serum polar metabolites, which ean predictassociate with the need for a CT scan. Additionally, serum metabolites can also predict the nature of the brain injury. These metabolite markers may prevent unnecessary CT scans, thus reducing the cost of diagnostics and radiation load.

Key Words: Metabolism, Traumatic Brain Injury, CT Scanning, Human Studies, Biomarkers 
1

2

3

4

5

6

7

8

9

\section{Introduction}

The diagnosis of traumatic brain injury (TBI) in the emergency room often relies upon a clinical neurological exam and neuroimaging. (1) The ability to more rapidly predict which patients will require $\mathrm{CT}$ imaging following a TBI is a long-standing problem and remains a key goal in the ability to triage patients upon admission.(2)

Previous efforts have focused on quantifying proteins such as S100 calcium-binding protein $\beta$ (S100ß), (3) glial fibrillary acidic protein (GFAP), (4) and ubiquitin C-terminal hydrolase-L1 (UCH-L1)(5) in the blood. Whilst studies have demonstrated the ability of these proteins to generate models which can predict the need for CT scan with reasonable areas under the curves (AUC $=0.78 \mathrm{~S} 100 \beta$, (6) $0.84 \mathrm{GFAP}$, (6) $0.73 \mathrm{UCH}-\mathrm{L} 1(5)$ ), the authors have not demonstrated the predictive power of these models using independent data sets. $\mathrm{S} 100 \beta$ is probably the most studied protein biomarker for TBI. However, it is not specific to brain or TBI $(7,8)$ : the levels of circulating $\mathrm{S} 100 \beta$ have been shown to vary after exercise (9) and patients who have sustained polytrauma where adipose tissue and bone are affected are known to exhibit high levels of S100ß. $(10,11)$ Recently however, GFAP, a protein expressed in the astrocytes within the CNS (12) has been shown to be a better predictor of the need for CT imaging. However, these studies did not contain an independent validation cohort, which is an essential component when employing diagnostic or prognostic models due to the risk of overfitting of the predictive model if only one dataset is used (13). Additionally, these proteins appear to have better predictive power in patients with more severe TBI $(1,14,15)$. This observation could well reflect the fact that changes in these proteins happen in the CNS behind the blood-brain-barrier (BBB) or, in the case of GFAP changes in the BBB itself, which will become more permeable in more severe cases $(16,17)$, thus altering the levels of brain derived proteins in the blood. Furthermore, GFAP may well also be non-specific to TBI, because Schwann cells in the periphery are known to increase 
GFAP production following injury $(18,19)$. Indeed, our recent study showed that GFAP and UCH-L1 were unable to separate patients with CT-negative mild TBI (mTBI) from patients with orthopaedic injuries. (20)

To date metabolic studies in TBI have mainly focused on trying to measure the changes in metabolism in the brain using a combination of techniques such as Magnetic Resonance Spectroscopy (MRS), Positron Emission Tomography (PET), and measuring polar metabolites from brain microdialysates. (21) These techniques are expensive and/or invasive and not widely available to all TBI patients especially those with less severe injuries. Previous attempts at looking at metabolic changes in the blood have targeted a very small number of molecules such as glucose and lactate. (22) To date the application of modern untargeted metabolomic approaches to study TBI have been sparse- and to our knowledge there are no studies exploring the role of metabolomics in detecting the need for a CT scan following a TBI. (23) Serum metabolomics is a powerful tool for non-invasively identifying biomarkers in disease. (24-26) We have previously shown that the severity of TBI and the eventual outcomes are associated with the changes in serum metabolome. (21) Furthermore, attempts at generating diagnostic models in animal models of TBI (27) have been reported using a combination of molecular lipids and polar metabolites measured from serum. However, most of these studies lacked a suitable validation cohort and therefore, their use as a diagnostic tool is currently still limited. Here we use gas chromatography, which covers $\underline{\text { small polar metabolites such as sugars, amino acids and free fatty acids in an untargeted }}$ approach $(28,29)$ to screen for new metabolites which can predict the need for a CT following a TBI.

The principal aim of this study was to assess whether serum metabolomics can be used distinguish between patients with traumatic intracranial findings and negative findings on head CT. We assessed the use of metabolomics for the whole range of TBI severities and 
then examined just the mild TBI cases. using logistic regression modelling. We then compared the metabolomics models to the results from protein assays acquired from the blood and explored if together they have an additive effect in terms of predicting the need for a head CT. In addition to this primary aim, we also assessed if serum metabolomics can distinguish between diffuse CT pathology and mass lesions, and whether we could predict the need for surgical intervention of mass lesions. Finally, we explore the potential origins of the metabolite changes by comparing the metabolite changes in serum to that of metabolite changes in the cerebral microdialysis of the same individuals and comparing it to an appropriate control group. 


\section{Methods}

\section{Ethics Statement}

All protocols used in this study were approved by South-West Finland Hospital District Research Ethics Committee, the Cambridgeshire 2 Research Ethics Committee, and the Norfolk Research Ethics Committee depending which country the samples were collected in. Oral and written consent was obtained from either the patient or their next of kin. All patients received treatment based on local standards and current international guidelines and recommendations. $(28)(30)$

\section{Sample selection and clinical details}

The patients in this study were a subgroup of individuals from our previously published cohort. (21). The patients were recruited as part of the EU funded TBI care (Evidence-based Diagnostic and Treatment Planning Solution for Traumatic Brain Injuries) project. Patients were included if they were older than 18 years (16 in the UK) and had a clinical diagnosis of TBI and indications on a head CT according to the National Institute for Health and Care Excellence (NICE) criteria. Patients were excluded if the injuries were blast-induced or penetrating injury, chronic subdural hematoma, pre-existing brain injuries or conditions, which caused non-independent living, TBI or suspected TBI two weeks prior to recruitment, non-native speaker, and no if no consent was obtained.

Out of 620 screened patients with suspected TBI, total 203 adults with acute TBI and 40 patients with orthopedic injuries with no known head trauma were recruited at Turku University Hospital (Turku, Finland). With serum samples available for comprehensive metabolomics profiling, 144 patients with verified TBI and 28 patients with orthopedic injury and carefully excluded TBI were included in the discovery cohort. The causes for not being recruited among patients with suspected TBI was available for 568 patients and the 
commonest reasons for exclusion were: research staff was unable to recruit $(\mathrm{n}=191,34 \%$ ), age less than 18 years $(n=82,14 \%)$, and uncertain diagnosis for TBI $(n=75,13 \%)$. 41 orthopedic controls were recruited from Addenbrooke's Hospital (Cambridge, UK). With serum samples available for comprehensive metabolomics profiling, 188 patients with verified TBI and 27 patients with orthopedic injury and rigorously excluded TBI were included in the validation cohort. The commonest reasons for exclusion among patients with suspected TBI were: research staff was unable to recruit $(n=352,31 \%)$, patient being too frail for reliable follow-up $(n=275,25 \%)$, and pre-existing disease $(n=219,20 \%)$.

The patient demographic data is summarized in Table 1-and the flow chart of the study process is presented in Figure 1..

The blood samples used in this analysis was collected within $12 \mathrm{~h}$ of admission to hospital by research nurses-. and other study personnel. There are some patients who were found unconscious and transferred to hospital and patients who sustained mTBI and sought for medical attention with latency. In these patients, the exact time of injury is unknown. The serum was allowed to clot for $30 \mathrm{mins}$ at $4{ }^{\circ} \mathrm{C}$. The blood was then centrifuged $(10 \mathrm{~min}$, $10,000 \mathrm{rpm}, 4^{\circ} \mathrm{C}$ ). All blood samples were stored at $-70^{\circ} \mathrm{C}$ in aliquots to prevent the need for freeze-thaw cycles. Cerebral microdialysis were collected from a subsection of the individuals in Cambridge $(\mathrm{n}=12)$ who had had a sTBI. The samples were collected using a

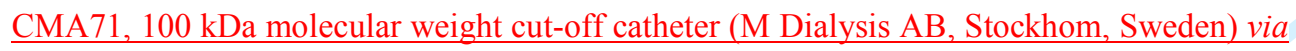
a cranial device (Technicam, Newton Abbot, UK) with a perfustion rate of $0.3 \mathrm{ml} / \mathrm{min}$ using a CMA106 pump (M Dialysis AB). The fluid consisted of CNS perfusion fluid (M Dialysis $\underline{\mathrm{AB})}$. The CT images were graded using the Marshall eriteriaclassification $(29)(31)$ and this was used as a classifier for the four aims in this study: 
Aim 1: Marshall Grade 1 (CT negative) vs. Marshall Grade 2-6 (CT positive)

Aim 2: Marshall Grade 2-4 (diffuse injury) vs. Marshall Grade 5-6 (mass lesion)

Aim 3: Grade 1-4 and 6 (conservative treatment) vs. Grade 5 (surgical treatment)

Aim 4: Grade 5 (operated mass lesion) vs. Grade 6 (non-operated mass lesion)

Aim 1 and 2 were considered primary aims and Aim 3 and 4 secondary. Marshall

classification was chosen, because it can be appropriately used for the aforementioned patient group division and to address the clinical questions. The CT scans were analyzed by

neuroradiologists and double-read by a senior neurosurgeon (JPP) and a neurologist (OT).

The samples were collected and processed as previously described. (21) 


\section{Metabolomics}

Metabolomic analysis was carried out with comprehensive two-dimensional gas chromatography (Agilent 6890, Agilent Technologies, Santa Clara, CA) combined with timeof flight mass spectrometry (Pegasus GC-HRT, Leco Corp., St. Joesph, MI, USA) as described. (21) Briefly, $10 \mu \mathrm{l}$ of internal standards (C17:0 186.5 mg/L, deuterated valine (37 $\mathrm{mg} / \mathrm{L}$, and succinic acid-d4 (63 mg/l)) were added to serum samples $(30 \mu \mathrm{l})$ or microdialysate samples $(100 \mu \mathrm{l})$. The protein was precipitated by the addition of $400 \mu \mathrm{l}$ of $\mathrm{MeOH}$ and the resultant mixture was vortexed for 2 mins. The samples were then centrifuged (7800 g, $5 \mathrm{~min}$, room temperature) prior to being left to settle $\left(30\right.$ mins, $\left.-20^{\circ} \mathrm{C}\right)$. The supernatant was then removed and evaporated to dryness under a flow of $\mathrm{N}_{2}$ gas at $45{ }^{\circ} \mathrm{C}$. The metabolites underwent a two-step derivatization, initially methoxyamine hydrochloride $(98 \%$ in pyradinepyridine) was added and the resultant mixture was incubated for $1 \mathrm{~h}$ at $45^{\circ} \mathrm{C}$. Next N-methyl-N-trimethylsilyltrifluoroacetamide $(25 \mu \mathrm{L})$ was added and the sample incubated for a further $1 \mathrm{~h}$ at $45^{\circ} \mathrm{C}$. Just prior to injection a series of alkane standards (n-alkanes, $25 \mu \mathrm{L}, \mathrm{c}$ $=8 \mathrm{mg} / \mathrm{l})$ and an injection standard (4,4'-dibromooctafluorobiphenyl, $50 \mu \mathrm{l}, \mathrm{c}=10 \mathrm{mg} / \mathrm{l}) \mathrm{were}$ added in order to calculate the retention index of each metabolite. The derivatization and sample injection was automated by using a multipurpose sampler (Gerstel, Mülheim an der Ruhr, Germany). The samples were separated using a $10 \mathrm{~m}$ x $0.18 \mathrm{~mm}$ inner diameter (ID) Rxi-5 ms (Resteck Corp., Bellefonte, PA, USA) column with a film thickness of $0.18 \mu \mathrm{m}$ as a first column and a $1.5 \mathrm{~m}$ x $0.1 \mathrm{~mm}$ ID BPX-50 (SGE Analytical Science Austin, TX, USA) column with film thickness of $0.1 \mu \mathrm{m}$ as a second column. A deactivated retention gap column (phenyl methyl, $1.5 \mathrm{~m} \times 0.53 \mathrm{~mm}$ ID) was used as a guard column. $1 \mu \mathrm{l}$ of samples was injected in splitless mode $\left(240{ }^{\circ} \mathrm{C}\right)$. The splitless mode lasted for $90 \mathrm{~s}$ with high purity helium (Aga, Espoo, Finland) used as the carrier gas. Constant pressure was used throughout 
$\left(276 \mathrm{kPa}\right.$ ). The $\mathrm{GC}$ oven was programed as so: $50{ }^{\circ} \mathrm{C}$ (isothermal for $2 \mathrm{~min}$ ), then $7{ }^{\circ} \mathrm{C} / \mathrm{min}$ until $240{ }^{\circ} \mathrm{C}$ and then $25{ }^{\circ} \mathrm{C} / \mathrm{min}$ until $300{ }^{\circ} \mathrm{C}$ (isothermal for $3 \mathrm{~min}$ ). The second coloumncolumn was held at a temperature $20{ }^{\circ} \mathrm{C}$ higher than the first, however, the programed temperature changes remained the same. The transfer line was maintained at 260 ${ }^{\circ} \mathrm{C}$ and the source temperature at $200{ }^{\circ} \mathrm{C}$. The modulation time was $4 \mathrm{~s}$. The mass range 45 to $700 \mathrm{amu}$ with $100 \mathrm{spectra} / \mathrm{s}$ were measured using electron impact ionization $(70 \mathrm{eV})$.

All raw data processing was performed in ChromaTOF v4.32 (LECO Corporation, St. Joseph, MI). Peaks were selected automatically using the inbuilt spectral deconvolution using a peak width of $0.2 \mathrm{~s}$. Any peak with a signal to noise ratio $>100$ were rejected. For the majority of compounds the peak areas from the total ion chromatogram was used. However, if the metabolites waswere quantified directly in the ChromaTOF software then the peak areas of the selected $m / z$ were used.

The output text files from ChromaTOF were then imported into the in housed developed | software Guineu (30).(29). This software was used for aligning and normalizing the compounds for further analysis. Since the second column is so short its contribution to the RI was considered negligible so the RI could be directly compared to spectral libraries. The spectra were aligned in the first dimension using these RI values. To further align the spectra retention times and spectra were used as a second dimension. Following alignment, the following criteria was used to filter the data to ensure positive identification: (1) spectral similarity $>850$, (2) the maximum difference between RI values and literature values $<25$. Additionally each metabolite had to appear in $70 \%$ of the samples run in in each of the four original study groups. (21) This resulted in a total of 465 metabolites. All literature values were obtained from the NIST 2008 ass Spectral Library. 


\begin{abstract}
Further identification of unknown metabolites was performed using $\mathrm{GC} \times \mathrm{GC}$ equipped with high--_resolution MS system and both electron impact and chemical ionization in conjunction with the GOLM database. (31) Metabolites that appeared in less than $70 \%$ of the samples were excluded from the subsequent analysis.(32) Metabolites that appeared in less than $70 \%$ of the samples were excluded from the subsequent analysis. In total 465 metabolites were detected using these criteria. Any metabolites that were identified as drugs such as propofol or ibuprofen were excluded from the data set as described previously. (21) Additionally, downstream metabolites of these compounds were excluded by examining which other metabolites highly correlated to the drug. This resulted in a total number of 455 metabolites, which were used in subsequent analysis.
\end{abstract}

The orders of both sample preparation and analysis were randomized, and a set of controls samples (pooled serum samples), standards and blank samples (solvent blank) was analyzed together with the samples. The day-to-day variation of internal standards added to all samples was on average $17.3 \%$ and $12.3 \%$ in the discovery and validation sets, respectively. The dayto-day variation in control serum samples $(\mathrm{n}=31)$ of the quantified metabolites was $18.0 \%$ and $9.2 \%$ in the discovery and validation sets, respectively. The linear range of the method is from 0.1 to $120 \mathrm{ng} / \mu$ l for quantified metabolites, with limit of quantification (LOQ, S/N 10) ranging from 0.005 to $0.1 \mathrm{ng} / \mu 1$. (3233)

Field Code Changed

Imaging predictions - logistic regression modelling

$\underline{\text { In order to generate a classification model, } 1000 \text { cross-validated logistic regression models }}$ were generated from the Turku (discovery cohort) data. The models were cross-validated by selecting the best model from a group of 10 where $10 \%$ of the discovery data was withheld and used as a prediction set. The models were selected based on the area under the curve 
(AUC) of the resultant receiver operator curve (ROC). Once the 1000 models had been generated from the Turku data, the models were validated with the data from Cambridge. The need for a discovery and validation cohorts prevents the overfitting of the data as commonly seen in multivariate modelling of the data. To select the optimal combination of metabolites for the linear regression, we introduced metabolites in an iterative manner starting with all metabolites that had a $\mathrm{Q}$ value $<0.05$. To select the base model for the next iteration, the model with the highest independently validated (using the Cambridge data) AUC was selected. If the AUCs were the same for multiple metabolites, all these were selected for the next iteration.

Protein analysis

GFAP and UCH-L1 were measured using a Randox biochip (Randox Laboratories Ltd, Crumlin, Country Antrim) as described elsewhere. (20)

\section{Statistical Analysis}

The protein and metabolite data was processed as described previously. (21) The statistical analysis was performed in MATLAB 2016a (Mathworks Inc., Natick MA) using the PLS toolbox 8.1 (Eigenvector Research Inc., Manson, WA) or GraphPad Prism 7.03 (GraphPad Software Inc. La Jolla, CA).

For metabolite and protein values that were marked zero, we imputed a value, which was equal to half the minimum for that analyte across all the samples. In order to correct for the non-normally distributed data, all values were log transformed prior to further analysis. For each of the four aims in the study, a student's Student's t--test was performed to identify the metabolites, which differed significantly between the two groups. False Discovery Rates (FDR) were calculated to correct for multiple comparisons. (33)(34) Q values less than 0.05 
1

2

3

4

5

6

7

8

9

10

11

12

13

14

15

16

17

18

19

20

21

22

23

24

25

26

27

28

29

30

31

32

33

34

35

36

37

38

39

40

41

42

43

44

45

46

47

48

49

50

51

52

53

54

55

56

57

58

59

60

were considered significant. Differences in protein and metabolite concentrations were assessed with Welch's corrected t-test due to the differences in variances between the groups.

The regression curve was calculated by linear regression and the residual values were plotted on a separate figure. Metabolites were deemed to be outside the regression model if the residual value was greater than two standard deviations.

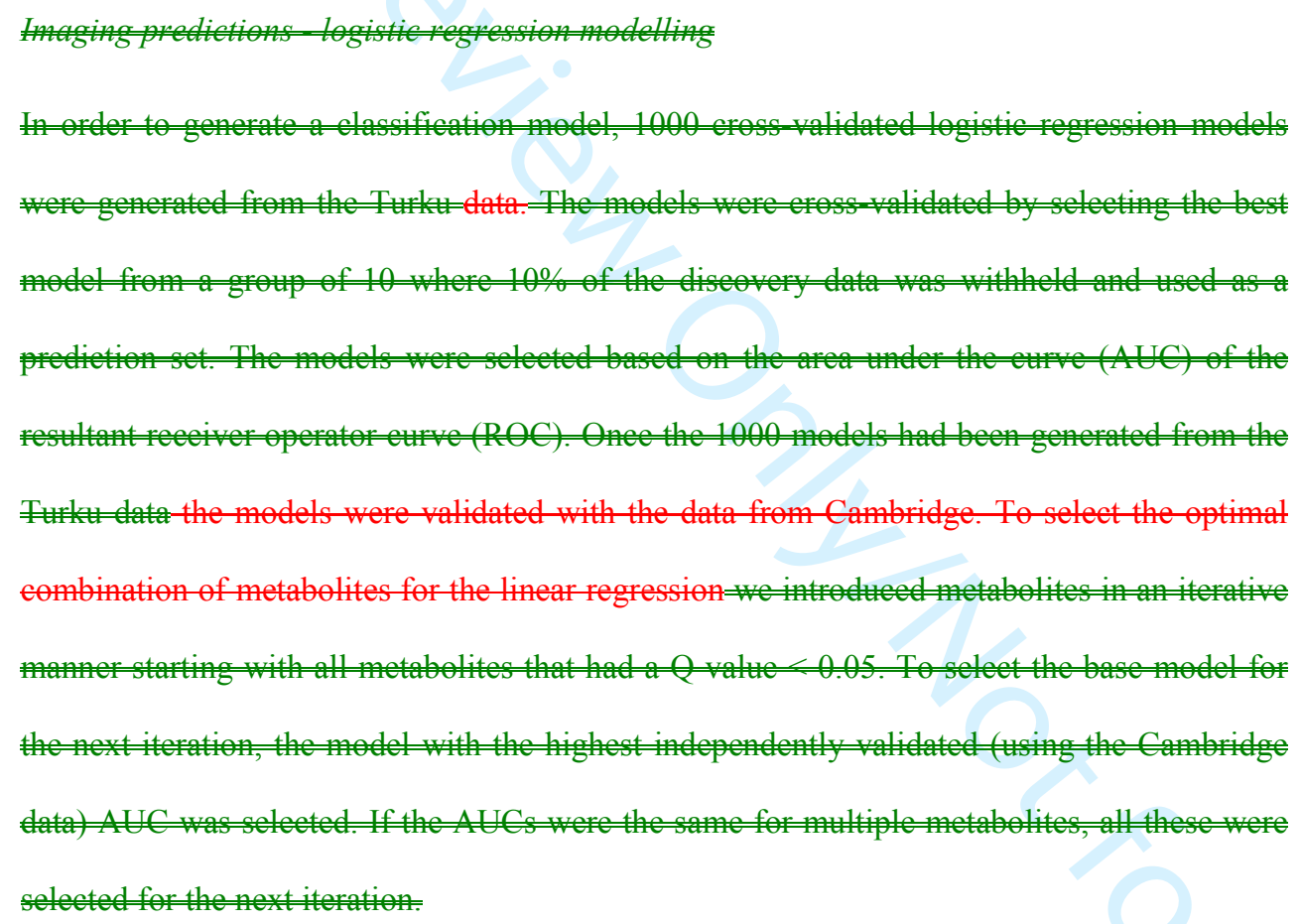




\section{Results}

\section{Patient Inclusion}

We included all patients $(\mathrm{n}=210)$ who had sustained TBI (regardless of severity) from both from-Turku, Finland $(n=144)$ and Cambridge, UK $(n=66)$. TheThe flow chart for patient selection is shown in Figure 1. The serum metabolite levels measured from orthopedic controls from both Turku $(n==28)$ and Cambridge $(n=27)$ were used in the brain microdialysate analysis to normalize the metabolite concentrations. These patients had other peripheral injuries but with no evidence of head trauma. (21) For a flow chart of patient selection please see Figure 1Serum metabolites associate with changes in CT imaging1

Serum metabolites can predict the need for a CT scan in a multi-center setting

WeUsing a univariate approach (Welch t-test with FDR correction) we identified 36 metabolites that differed between the patients who had traumatic intracranial findings (CT positive) as compared to patients with negative CT findings (CT negative) (Table 2). Next, we built a logistic regression model to predict which patients with TBI would be CT positive. The iterative process resulted in a predictive model (sensitivity $=0.73$, specificity $=0.64$; Figure 2a), which utilized six metabolites (scatter plots shown in Figure 2b-h), including two amino acids, three sugar derivatives and one ketoacid. The model had good predictive accuracy for bothin the discovery cohort, Turku (AUC $=0.77 ; 95 \%$ CI: 0.69-0.87) and the validation group, Cambridge data $(\mathrm{AUC}=0.73 ; 95 \% \mathrm{CI}: 0.73-0.74)$, thus suggesting that the metabolites are more reliable in a multi-center setting as compared to proteins, when predicting the need for a $\mathrm{CT}$ sean.). Next, we hypothesized that a combination of metabolites and proteins might furnish a more accurate prediction model. When the protein data was included in the logistic regression model, the predictive accuracy did not improve to a 
meaningful extent: Turku, discovery cohort, $\mathrm{AUC}=0.78$ (95\% CI: 0.69-0.87), Cambridge, validation cohort, $\mathrm{AUC}=0.73(95 \% \mathrm{CI}: 0.73-0.74)$.

\section{Mild TBI}

We sought to build logistic regression model to classify, which patients require a CT scan using only the patients who had a mTBI (GCS $\geq 13$ ). Initially, we used the same optimized list of metabolites identified across the whole spectrum of TBI (Figure 3a). The resulting model had good accuracy prediction in the Turku dataset (AUC $=0.73 ; 95 \%$ CI: 0.64-0.68) but did not validate in the Cambridge dataset ( $A U C=0.57 ; 95 \% \mathrm{CI}: 0.57-0.57)$. Therefore, we performed the univariate analysis using only the mTBI patients. This only furnished three metabolites that changed in patients who required a CT sean. The optimal combination of metabolites resulted in a logistic regression model with good accuracy in the Turku-data $(\mathrm{AUC}=0.75 ; 95 \% \mathrm{CI}: 0.67,0.86)$, but it validated poorly in the Cambridge samples (AUC = $0.53 ; 95 \%$ CI: $0.53,0.53)$.

GFAP and UCH-L1 validate poorly in a multi-center setting when predicting the need for a CT scan

We measured GFAP and UCH-L1 in two independent cohorts acquired from Turku (discovery cohort, Finland) and Cambridge (validation cohort, UK). There were six samples from Turku and three from Cambridge, which did not have protein data available. Using a univariate approach (Welch's t-test) there were clear increases in both GFAP $\left(p_{\text {turku }}=0.0002\right.$; $\left.p_{\text {cambridge }}=0.0004\right)$ and UCH-L1 $\left(p_{\text {turku }}=0.0094\right)$ in patients who had abnormalities on the CT scan, when compared to TBI patients with no CT pathology (Figure 3 A-D). The one exception to this were the UCH-L1 levels in the Cambridge cohort which did not differ between the groups $\left(p_{\text {cambridge }}=0.1823\right)$. Using the logistic regression modelling in the Turku 
cohort, it was possible to generate models using GFAP (AUC $=0.73$; 95\% CI: 0.64-0.83), $\mathrm{UCH}-\mathrm{L} 1(\mathrm{AUC}=0.71 ; 95 \% \mathrm{CI}: 0.62-0.85)$ or a combination of both $($ Figure $3 \mathrm{E}$ AUC $=0.73$; 95\% CI: 0.62-0.86), that could predict abnormalities on a CT scan (Marshall Grade > 1).

When the Cambridge data was used for validation, the models did not perform as expected, with GFAP (Figure 3E AUC $=0.64 ; 95 \%$ CI: 0.64-0.64), UCH-L1 (AUC $=0.58 ; 95 \%$ CI: 0.58-0.58) and the combination model (AUC $=0.64 ; 95 \% \mathrm{CI}: 0.63-0.64)$ all giving poorer predictive accuracy.

\section{Serum metabolites can be used to predict other CT based features}

When comparing diffuse lesions (Marshall Grade $=2-4)$ with mass lesions $($ Marshall Grade $=$ 5-6), we identified six metabolites (Table 3). No significant metabolites were identified when comparing surgically operated lesions (Marshall Grade $=5$ ) with all other patients with TBI (Marshall Grade 1-4 and 6). Finally, when comparing patients who had mass lesions in the CT, five metabolites were identified when patients who had surgically operated mass lesions were compared to patients who were not treated surgically (Table 4).

Given that only a limited number of metabolites changed significantly between the groups for aimsaim 2-and 4, we built linear regression models for these. When classifying the CT positive TBI patients into those who had diffuse injuries compared to mass lesions (Aim 2), the resulting model performed well with the Turku data $\mathrm{AUC}=0.87$ (95\% CI: 0.75-0.94) and cross-validated to some degree in the Cambridge data AUC $=0.68$ (95\% CI: 0.68-0.69) (Figure 4a). The logistic regression model comprised three metabolites (Figure 4b-d), an unknown phenolic compound, isovalerylglucuronide and 2-hydroxybutyric acid. When comparing surgically operated mass lesions to non-surgically operated mass lesions, the model had good predictive accuracy in the Turku dataset AUC $=0.84$ (95\% CI: 0.70-0.90). 
However, this model did not validate as well when applied to the Cambridge dataset AUC $=$ 0.60 (95\% CI: 0.60-0.61). This logistic regression model comprised two metabolites, a sugar derivative and octanoic acid.

Metabolites measured in the serum associate with levels measured in brain microdialysates (BMD)

In our previous study the levels of metabolites were determined in the brain microdialysate (n $=12)(21)$. The results from this analysis are reproduced here (Figure 5a). This original study compared the metabolite levels to orthopedic controls. (21) Since the metabolites used in the linear prediction models differed from those that associated with the severity of TBI, a single regression line was calculated from the data as opposed to the two generated in the previous study. (21) In order to understand the origins of the metabolite changes in the present study, we calculated the vertical distance (residual, e) between the predicted value and the measured value for each metabolite (Figure $5 b)$. The sugar derivatives inositol $(\mathrm{e}=0.018)$, ribonic acid ( $\mathrm{e}=0.079)$, pentitol 3-desoxy $(\mathrm{e}=-0.316)$, and isovalerylglucuronide $(\mathrm{e}=0.355)$ had low residual values, demonstrating that the metabolite concentrations are closely associated between BMD and serum. However, the unknown phenolic compound $(\mathrm{e}=3.49)$ did not fit with the regression line. Additionally, there were two metabolites, acetoacetic acid and an unknown amino acid, that were not detected in the BMD at all. 


\section{Discussion}

There is an unmet clinical need to distinguish if a patient requires a head CT scan following

TBI. Here we demonstrate the effectiveness of circulating metabolites in stratification of patients based on their CT imaging. Our results show that metabolites can generate better cross-validated predictions when compared to protein measures. We have identified a metabolite panel, which can discriminate between diffuse brain injury and mass lesions as observed in the CT scan.

One of the key strengths of this study is the use of two independent cohorts, which enables us to cross-validate the predictive models in a multi-center setting. We were surprised by the poor performance of the protein measurements duringin a multicenter setting compared to the eross - alidationmetabolomics results, especially given the previously published data which showed some promise. $(1,4-6)$ The primary limitation of the earlier studies was the lack of external validation and potentially the wide variety of analytical platforms used. The method in this study was a novel chip based assay. Our results suggest that even though the GFAP and UCH-L1 concentrations increase following the injury, they do not do so in a consistent enough for logistic regression modelling. There are many possible explanations why metabolites in the blood can be more suitable to model the TBI-related changes in the brain as proteins. One of the key factors is the relative size difference between metabolites (100$1500 \mathrm{Da})$ and proteins $(10,000-100,000 \mathrm{Da})$. Metabolites can cross the BBB more readily following the injury for several reasons, ranging from BBB breakdown (34)(35) to subtle changes in astrocytes in response to inflammatory stimuli (35)(36) or alterations in the hypoxia-inducible factor-1 $\alpha$ pathway and elevated expression of aquaporin-4. (36)(37) The changes in the proteins, especially GFAP, could arise from changes to the BBB itself as opposed to changes within the brain parenchyma. It could be that the protein measures are more sensitive to differences in sample handling. (37-3938-40) This limitation could in 
principle be solved by imposing stricter standard operating procedures (SOPs) in the clinic. The robustness of a predictive model to sample handling is a key feature when developing a clinically relevant tool, $(38) \underline{(39)}$ as the wider the implementation, the harder it is to achieve compliance with complicated SOPs. Aside from proteins there is a set of clinical guidelines utilized in Europe assessing the need for a CT scan based on clinical data. Unfortunately, it was impossible to assess these patients usingClinical CT prediction tools, such as the UK NICE Criteria, can provide useful bases for assessing the need for a CT scan following TBI (40) due to the scareity of iterative GCS data in the emergency room. A simple blood test, which with further development a metabolomies assay could be, removes the need for gathering such clinical data, which can be problematic(41). However, such schemes are imperfect, and could be improved by a simple blood test, which could be developed with further work on a metabolomics assay. However, a larger study directly comparing the NICE guidelines to a metabolomics assay would need to be performed to fully assess the clinical utility of a metabolomics based assay.

Given that we are determining the serum metabolites following a brain injury, it is challenging to identify the sources of the variation observed. All the sugar-related metabolites, pentitol 3-desoxy, inositol, isovalerylglucuronide, and ribonic acid, which generated predictive models fitted the correlation model. All these sugar derivatives increased in concentration when there was detectable pathology on a CT scan or a presence of a mass lesion suggesting that the source of these changes originate from the brain. There have been several studies that demonstrate altered glucose metabolism $(22,41 \underline{42})$ following TBI, which could also explain the increase of sugar derivatives in the circulation.

2-aminobutyric acid is another metabolite fitting the correlation model. Its concentration was lower in patients with detectable CT features. These reduced levels in the serum could indicate increased oxidative stress (42) in the brain.(43) in the brain. Elevated oxidative stress 
markers have been observed in the CSF of patients with severe TBI $(43)(44)$ reinforcing the probability that changes in 2-aminobutyric acid originated within the CNS. Some

We speculate that the unknown phenolic compound may be propofol, or one of its metabolites. Propofol is an intravenous anaesthetic which is commonly used for sedation in patients with moderate to severe TBI who require tracheal intubation and mechanical ventilation for airway protection and control of ventilation(45). It is also important to recognize that propofol, which is highly lipophilic, is solubilized in an oil in water emulsion which contains $100 \mathrm{mg}$ soya bean oil per milliliter(46). The additional lipid load provided by this formulation would only have been present in patients with more severe TBI, and hence a greater likelihood of having a positive CT, and consequently represents a potential confound. Other potential explanations also need to be considered. While starvation ketosis cannot be excluded, this seems an unlikely cause after less than a day's starvation. ThisFurther, the change in systemic levels potentially suggests a source other than the brain, of metabolite changes following TBI. This is not unexpected, as clear evidence exists that brain injuries result in changes in the periphery. Immediately after the injury there is an induction of acute phase proteins in the liver. $(44,45 \underline{47,48})$ Additionally, there are modulation of proteins, such as peroxisome proliferator-activated receptor alpha, (4649) which play a key role in lipid homeostasis. One caveat about the metabolites absent in the BMD is that the metabolite concentration compared to serum is very low. However, highly lipophilic metabolites may 
The two metabolites, not observed in the BMD, could be there but at low levels. They still not fit the correlation model, thus indicating a non-brain derived origin to the change in concentration of these metabolites. From these three metabolites only acetoacetic acid can be identified. It is a ketone body produced in the liver. (17) $(50)$ These act as an energy source following injury. (48)(51) Fasting to induce ketosis has been shown to have a neuroprotective effect following TBI. (49)(52) The other non-identified metabolites make it hard to interpret their biological function. It would appear that changes in these metabolites are not brain derived.

There are several limitations to this current study. Such as the difference between the two mTBI cohorts. First, though the recruitment process with identical inclusion and exclusion criteria of TBI care consortium was carefully planned while preparing the study protocol, the representativeness of included population varies between the Turku and Cambridge cohorts. This may partially be due to fact that varying recruitment resources and information transfer about the inbound patients at the emergency departments. In Cambridge, only $17 \%$ of the patients with suspected TBI were included in the study, while in Turku, $33 \%$ were included. Both of the institutions are university hospitals with a distinction that Turku University Hospital serves as a both primary emergency center and referral center whereas Addenbrooke's Hospital serves as a tertiary referral center. This might have an effect on the characteristics of trauma patients admitted.

Second, the mechanism of injury and the outcome as measured by the extended Glasgow Outcome Scale were different in the two cohorts (21) and the patients were older and had sustained more severe injuries based on the imaging findings especially in mTBI group in Turku cohort. The difference between two cohorts is presumably the reason why GFAP and 
UCH-L1 validated poorly in Cambridge cohort highlighting the problems of these protein biomarkers in acute diagnostics in cases of milder TBIs.

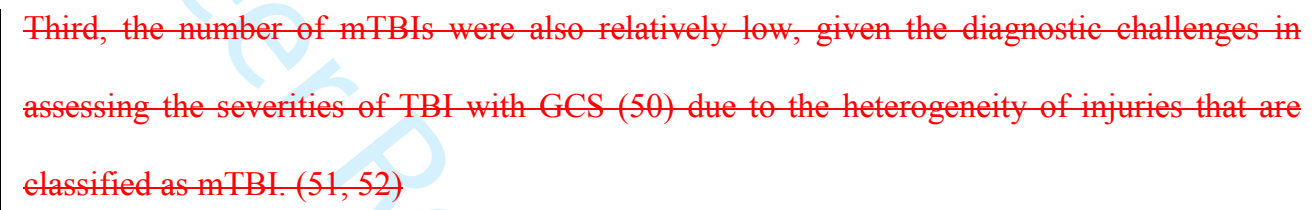

In conclusion, it is likely that there is a notable variation in the representativeness, preexisting health problems based on the differences in cohort demographics and injury characteristics between the study cohorts. Hence the Turku cohort, particularly the mTBI group,-is older and more seriously injured. To fully explore these patients, a larger study is necessary.

The metabolites identified from the full spectrum generated a model that internally crossvalidated in the Turku cohort, which suggests that metabolites will be the best source of providing CT predictive models in the future. Low patient numbers was a key factor in the lack of accuracy or significant metabolites when addressing the need for surgery following a mass lesion. The fact that there are no clinical guidelines, about when to operate on mass lesions and it can slightly vary between the centers and within the centers is a further issue. This highlights the need for better stratification tools to optimize and standardize the care following TBI. Interestingly, the metabolites that changed when comparing surgically treated to non-operated mass lesions, were octanoic and decanoic acids, which we had previously shown to associate with the severity of the injury. (21)

The mass spectrometry technology used in this study is a profiling research method, as opposed to a targeted assay, which provides broad analytical coverage and high sensitivity but is not fully quantitative and thus not suitable for applications in the healthcare setting. (53): The metabolites identified could be quantified using a targeted MS approach for 
1

2

3

4

5

6

7

8

9

selected metabolites. Such targeted assays and required infrastructure are readily available in typical clinical chemistry laboratories. The manual sample processing step which is required for the GC based profiling method can be simplified by either automating it or switching to a quantitative LC based method. The cost of a targeted MS assay is far less than a CT scan when considering interpretation cost. Such assays would reduce the cost of care following TBI and ensure patients only receive the X-ray radiation from a CT scan when required. Additionally, the fact that this was a global metabolite screen could contribute to the lower sensitivity and specificity observed in the metabolite model. A targeted screen focused on the metabolites identified in this manuscript should help increase this sensitivity and specificity. However, it is worth noting that the metabolites did outperform the proteins when employed in a multi-center setting which is more clinically relevant.

\section{Conclusion}

This study is the first to demonstrate the use of metabolomics in the serum for classifying patients with TBI who need a CT scan. We have demonstrated that the protein serum markers GFAP and UCH-L1 perform poorly in a multi-centre setting. Additionally, we have identified metabolites, which can predict whether the type of brainpatient has diffuse injury eecurredor mass lesion. The metabolite models could have an impact on the ability to provide personalized healthcare to patients with TBI and prevent unnecessary CT scans.

\section{Acknowledgements}

The work was supported by the EU FP7 project TBIcare (Project ref. 270259 to MO DM and OT), by the GE-NFL Head Health Challenge I Award (grant no. 7620 to MO and TH), by persenal EVO fundingGoverment's Special Financial Transfer tied to academic research in Health Sciences (Finland) (to JPP and RSKT.), and by personal grant from Emil Aaltonen Foundation and Finnish Brain Foundation (to JPP). Support for P.J.H. - National Institute for 
Health Research (NIHR) Research Professorship; NIHR Biomedical Research Centre (NIHR BRC) Cambridge. Support for K.L.H.C. - NIHR BRC Cambridge. This work was supported

We would like to thank all the study participants of TBI care for providing the samples used in this study. We would also like to thank Mark Van Gils who coordinated the TBI care project. Furthermore, we would like to thank Anna-Liisa Ruuskepää for technical assistance in metabolomics analysis. The authors would also like to thank research nurses Patricia Bertenyi, and Satu Timlin for their valuable contribution to this study.

\section{Author Contribution}

The study was designed by JPP, AMD, OT, TH, and MO. The study protocol was designed by TH and MO. Patients were recruited, clinical data collected, and blood samples by JPP, RSKT, AJK, HAS, AK, H-RM, JT, JPC, and JF. KLHC and PJH performed brain microdialysis studies. IM and $\mathrm{TH}$ acquired and processed the metabolomics analysis. $\mathrm{TH}$ identified the unknown metabolites identified by the modelling. AMD performed all the univariate and linear regression modelling. AMD and $\mathrm{MO}$ drafted the first versions of the manuscript. JPP, DM, OT and TH provided critical contributions. All authors reviewed edited and approved the final version of the manuscript.

\section{Conflicts of Interest}

AMD, JPP, TH, OT, and MO are all co-applicants on a patent relating to the use of metabolomics for the diagnosis and prognosis of mTBI. 


\section{References}

1. Posti, J.P., Takala, R.S., Runtti, H., Newcombe, V.F., Outtrim, J., Katila, A.J., Frantzén, J., Ala-Seppälä, H., Coles, J.P. and Hossain, M.I. (2016). The levels of glial fibrillary acidic protein and ubiquitin C-terminal hydrolase-L1 during the first week after a traumatic brain injury: correlations with clinical and imaging findings. Neurosurgery 79, 456-464.

2. Haydel, M.J., Preston, C.A., Mills, T.J., Luber, S., Blaudeau, E. and DeBlieux , P.M.C. (2000). Indications for Computed Tomography in Patients with Minor Head Injury. New England Journal of Medicine 343, 100-105.

3. Biberthaler, P., Linsenmeier, U., Pfeifer, K.-J., Kroetz, M., Mussack, T., Kanz, K.-G., Hoecherl, E.F., Jonas, F., Marzi, I. and Leucht, P. (2006). Serum S-100B concentration provides additional information fot the indication of computed tomography in patients after minor head injury: a prospective multicenter study. Shock 25, 446-453.

4. Pelinka, L.E., Kroepfl, A., Leixnering, M., Buchinger, W., Raabe, A. and Redl, H. (2004). GFAP versus S100B in serum after traumatic brain injury: relationship to brain damage and outcome. J. Neurotrauma 21, 1553-1561.

5. Papa, L., Lewis, L.M., Silvestri, S., Falk, J.L., Giordano, P., Brophy, G.M., Demery, J.A., Liu, M.C., Mo, J. and Akinyi, L. (2012). Serum levels of ubiquitin C-terminal hydrolase distinguish mild traumatic brain injury from trauma controls and are elevated in mild and moderate traumatic brain injury patients with intracranial lesions and neurosurgical intervention. Journal of Trauma and Acute Care Surgery 72, 1335-1344.

6. Papa, L., Silvestri, S., Brophy, G., Giordano, P., Falk, J., Braga, C., Tan, C., Ameli, N., Demery, J. and Dixit, N. (2014). GFAP out-performs S100B in detecting traumatic intracranial lesions on $\mathrm{CT}$ in trauma patients with mild traumatic brain injury and those with extracranial lesions. J. Neurotrauma 31, 1815-1822. 
7. Anderson, V.A., Catroppa, C., Haritou, F., Morse, S., Pentland, L., Rosenfeld, J. and Stargatt, R. (2001). Predictors of acute child and family outcome following traumatic brain injury in children. Pediatr. Neurosurg. 34, 138-148.

8. Torabian, S. and Kashani-Sabet, M. (2005). Biomarkers for melanoma. Curr. Opin. Oncol. $17,167-171$.

9. Koh, S.X. and Lee, J.K. (2014). S100B as a marker for brain damage and blood-brain barrier disruption following exercise. Sports Med 44, 369-385.

10. Pelinka, L.E., Toegel, E., Mauritz, W. and Redl, H. (2003). Serum S 100 B: a marker of brain damage in traumatic brain injury with and without multiple trauma. Shock 19, 195-200. 11. Thelin, E.P., Jeppsson, E., Frostell, A., Svensson, M., Mondello, S., Bellander, B.-M. and Nelson, D.W. (2016). Utility of neuron-specific enolase in traumatic brain injury; relations to S100B levels, outcome, and extracranial injury severity. Critical Care 20, 285.

12. Sofroniew, M.V. and Vinters, H.V. (2010). Astrocytes: biology and pathology. Acta neuropathologica 119, 7-35.

13. Bleeker, S., Moll, H., Steyerberg, E., Donders, A., Derksen-Lubsen, G., Grobbee, D. and Moons, K. (2003). External validation is necessary in prediction research:: A clinical example. Journal of clinical epidemiology 56, 826-832.

14. Papa, L., Akinyi, L., Liu, M.C., Pineda, J.A., Tepas III, J.J., Oli, M.W., Zheng, W., Robinson, G., Robicsek, S.A. and Gabrielli, A. (2010). Ubiquitin C-terminal hydrolase is a novel biomarker in humans for severe traumatic brain injury. Critical care medicine 38, 138.

15. Takala, R.S., Posti, J.P., Runtti, H., Newcombe, V.F., Outtrim, J., Katila, A.J., Frantzén, J., Ala-Seppälä, H., Kyllönen, A. and Maanpää, H.-R. (2016). Glial fibrillary acidic protein and ubiquitin C-terminal hydrolase-L1 as outcome predictors in traumatic brain injury. World neurosurgery $87,8-20$. 


\begin{abstract}
16. Prakash, R. and Carmichael, S.T. (2015). Blood- brain barrier breakdown and neovascularization processes after stroke and traumatic brain injury. Current opinion in neurology 28, 556-564.
\end{abstract}

17. Price, L., Wilson, C. and Grant, G. (2016). Blood-brain barrier pathophysiology following traumatic brain injury.

18. Garrison, C., Dougherty, P., Kajander, K. and Carlton, S. (1991). Staining of glial fibrillary acidic protein (GFAP) in lumbar spinal cord increases following a sciatic nerve constriction injury. Brain Res. 565, 1-7.

19. Mancardi, G., Cadoni, A., Tabaton, M., Schenone, A., Zicca, A., De Martini, I., Bianchini, D., Damiani, G. and Zaccheo, D. (1991). Schwann cell GFAP expression increases in axonal neuropathies. Journal of the neurological sciences 102, 177-183.

20. Posti, J.P., Hossain, I., Takala, R.S., Liedes, H., Newcombe, V., Outtrim, J., Katila, A.J., Frantzen, J., Ala-Seppala, H., Coles, J.P., Kyllonen, A., Maanpaa, H.R., Tallus, J., Hutchinson, P.J., van Gils, M., Menon, D.K. and Tenovuo, O. (2017). Glial Fibrillary Acidic Protein and Ubiquitin C-Terminal Hydrolase-L1 Are Not Specific Biomarkers for Mild CTNegative Traumatic Brain Injury. J. Neurotrauma.

21. Orešič, M., Posti, J.P., Kamstrup-Nielsen, M.H., Takala, R.S., Lingsma, H.F., Mattila, I., Jäntti, S., Katila, A.J., Carpenter, K.L., Ala-Seppälä, H., Kyllönen, A., Maanpää, H.R., Tallus, J., Coles, J.P., Heino, I., Frantzén, J., Hutchinson, P.J., Menon, D.K., Tenovuo, O. and Hyötyläinen, T. (2016). Human Serum Metabolites Associate With Severity and Patient Outcomes in Traumatic Brain Injury. EBioMedicine.

22. Glenn, T.C., Kelly, D.F., Boscardin, W.J., McArthur, D.L., Vespa, P., Oertel, M., Hovda, D.A., Bergsneider, M., Hillered, L. and Martin, N.A. (2003). Energy dysfunction as a predictor of outcome after moderate or severe head injury: indices of oxygen, glucose, and lactate metabolism. Journal of Cerebral Blood Flow \& Metabolism 23, 1239-1250. 
23. Wolahan, S.M., Hirt, D., Braas, D. and Glenn, T.C. (2016). Role of Metabolomics in Traumatic Brain Injury Research. Neurosurgery Clinics of North America 27, 465-472.

24. Dickens, A.M., Larkin, J.R., Griffin, J.L., Cavey, A., Matthews, L., Turner, M.R., Wilcock, G.K., Davis, B.G., Claridge, T.D.W., Palace, J., Anthony, D.C. and Sibson, N.R. (2014). A type 2 biomarker separates relapsing-remitting from secondary progressive multiple sclerosis. Neurology 83, 1492-1499.

25. Antila, K., Lötjönen, J., Thurfjell, L., Laine, J., Massimini, M., Rueckert, D., Zubarev, R.A., Orešič, M., van Gils, M. and Mattila, J. (2013). The PredictAD project: development of novel biomarkers and analysis software for early diagnosis of the Alzheimer's disease. Interface Focus 3, 20120072.

26. Suvitaival, T., Mantere, O., Kieseppä, T., Mattila, I., Pöhö, P., Hyötyläinen, T., Suvisaari, J. and Orešič, M. (2016). Serum metabolite profile associates with the development of metabolic co-morbidities in first-episode psychosis. Translational Psychiatry 6, e951.

27. Bahado-Singh, R.O., Graham, S.F., Han, B., Turkoglu, O., Ziadeh, J., Mandal, R., Er, A., Wishart, D.S. and Stahel, P.L. (2016). Serum metabolomic markers for traumatic brain injury: a mouse model. Metabolomics 12, 100.

28. Bullock, M.R. and PovlishockPosti, J.P., Dickens, A.M., Orešič, M., Hyötyläinen, T. (2007). Guidelines for the management ofand Tenovuo, O. (2017). Metabolomics profiling as a diagnostic tool in severe traumatic brain injury. Editor's Commentary. J. Neurotrauma 24, 2 p preceding S1Frontiers in neurology 8, 398.

29.29. Castillo, S., Mattila, I., Miettinen, J., Orešič, M. and Hyötyläinen, T. (2011). Data analysis tool for comprehensive two-dimensional gas chromatography/time-of-flight mass

spectrometry. Analytical chemistry 83, 3058-3067.

30. Bullock, M.R. and Povlishock, J.T. (2007). Guidelines for the management of severe traumatic brain injury. Editor's Commentary. J. Neurotrauma 24, 2 p preceding S1. 
31. Marshall, L., Marshall, S.B., Klauber, M., Van Berkum, C.M., Eisenberg, H., Jane, J., Luerssen, T., Marmarou, A. and Foulkes, M. (1992). The diagnosis of head injury requires a classification based on computed axial tomography. J. Neurotrauma 9, S287.

30.Castillo, S., Mattila, I., Miettinen, J., Orešič, M. and Hyötyläinen, T. (2011). Data analysis tool for comprehensive to-dimensional gas chromatography/time-of-flight mass spementr. Analyieal hemisty $83,3058,3067$.

3132. Kopka, J., Schauer, N., Krueger, S., Birkemeyer, C., Usadel, B., Bergmüller, E., Dörmann, P., Weckwerth, W., Gibon, Y. and Stitt, M. (2005). GMD@ CSB. DB: the Golm metabolome database. Bioinformatics 21, 1635-1638.

3233. Hartonen, M., Mattila, I., Ruskeepää, A.-L., Orešič, M. and Hyötyläinen, T. (2013). Characterization of cerebrospinal fluid by comprehensive two-dimensional gas chromatography coupled to time-of-flight mass spectrometry. J. Chromatogr. A 1293, 142149.

3334. Storey, J.D. (2002). A direct approach to false discovery rates. Journal of the Royal Statistical Society: Series B (Statistical Methodology) 64, 479-498.

3435. Shlosberg, D., Benifla, M., Kaufer, D. and Friedman, A. (2010). Blood-brain barrier breakdown as a therapeutic target in traumatic brain injury. Nature Reviews Neurology 6, 393-403.

3536. Chen, Y. and Swanson, R.A. (2003). Astrocytes and brain injury. Journal of Cerebral Blood Flow \& Metabolism 23, 137-149.

3637. Higashida, T., Kreipke, C.W., Rafols, J.A., Peng, C., Schafer, S., Schafer, P., Ding, J.Y., Dornbos III, D., Li, X. and Guthikonda, M. (2011). The role of hypoxia-inducible factor-1 $\alpha$, aquaporin-4, and matrix metalloproteinase-9 in blood-brain barrier disruption and brain edema after traumatic brain injury: laboratory investigation. Journal of neurosurgery $114,92-101$.
Formatted: English (U.S.), Do not check spelling or grammar

Formatted: English (U.S.), Do not check spelling or grammar 
3738. Mitchell, B.L., Yasui, Y., Li, C.I., Fitzpatrick, A.L. and Lampe, P.D. (2005). Impact of freeze-thaw cycles and storage time on plasma samples used in mass spectrometry based biomarker discovery projects. Cancer informatics 1 .

38ㅉ․ Jørgenrud, B., Jäntti, S., Mattila, I., Pöhö, P., Rønningen, K.S., Yki-Järvinen, H., Orešič, M. and Hyötyläinen, T. (2015). The influence of sample collection methodology and sample preprocessing on the blood metabolic profile. Bioanalysis 7, 991-1006.

3940. Hsieh, S.Y., Chen, R.K., Pan, Y.H. and Lee, H.L. (2006). Systematical evaluation of the effects of sample collection procedures on low-molecular-weight serum/plasma proteome profiling. Proteomics 6, 3189-3198.

40⒈ Hodgkinson, S., Pollit, V., Sharpin, C. and Lecky, F. (2014). Early management of head injury: summary of updated NICE guidance. BMJ: British Medical Journal (Online) 348.

41느. Clausen, F., Hillered, L. and Gustafsson, J. (2011). Cerebral glucose metabolism after traumatic brain injury in the rat studied by $13 \mathrm{C}$-glucose and microdialysis. Acta Neurochir. $153,653-658$.

4243. Soga, T., Baran, R., Suematsu, M., Ueno, Y., Ikeda, S., Sakurakawa, T., Kakazu, Y., Ishikawa, T., Robert, M. and Nishioka, T. (2006). Differential metabolomics reveals ophthalmic acid as an oxidative stress biomarker indicating hepatic glutathione consumption. Journal of Biological Chemistry 281, 16768-16776.

434․ Bayir, H., Kagan, V.E., Tyurina, Y.Y., Tyurin, V., Ruppel, R.A., Adelson, P.D., Graham, S.H., Janesko, K., Clark, R.S. and Kochanek, P.M. (2002). Assessment of antioxidant reserves and oxidative stress in cerebrospinal fluid after severe traumatic brain injury in infants and children. Pediatr. Res. 51, 571-578.

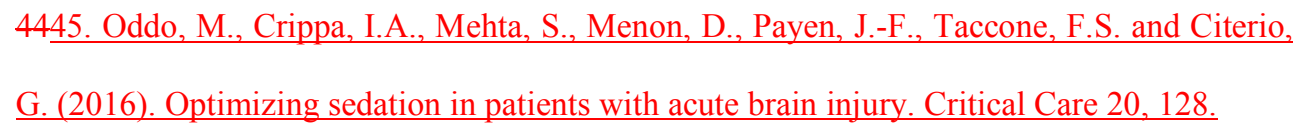




\section{https://www.accessdata.fda.gov/drugsatfda_docs/label/2008/019627s046lbl.pdf.}

47. Campbell, S.J., Hughes, P.M., Iredale, J.P., Wilcockson, D.C., Waters, S., Docagne, F., Perry, V.H. and Anthony, D.C. (2003). CINC-1 is identified as an acute-phase protein induced by focal brain injury causing leukocyte mobilization and liver injury. The FASEB Journal.

4548. Wilcockson, D.C., Campbell, S.J., Anthony, D.C. and Perry, V.H. (2002). The systemic and local acute phase response following acute brain injury. Journal of Cerebral Blood Flow \& Metabolism 22, 318-326.

4649. Dickens, A.M., Tovar, Y.R.L.B., Yoo, S.W., Trout, A.L., Bae, M., Kanmogne, M., Megra, B., Williams, D.W., Witwer, K.W., Gacias, M., Tabatadze, N., Cole, R.N., Casaccia, P., Berman, J.W., Anthony, D.C. and Haughey, N.J. (2017). Astrocyte-shed extracellular vesicles regulate the peripheral leukocyte response to inflammatory brain lesions. Science signaling 10.

4750. Newman, J.C. and Verdin, E. (2014). Ketone bodies as signaling metabolites. Trends Endocrinol. Metab. 25, 42-52.

4851. Prins, M.L. (2008). Cerebral metabolic adaptation and ketone metabolism after brain injury. Journal of Cerebral Blood Flow \& Metabolism 28, 1-16.

4952. Davis, L.M., Pauly, J.R., Readnower, R.D., Rho, J.M. and Sullivan, P.G. (2008). Fasting is neuroprotective following traumatic brain injury. J. Neurosci. Res. 86, 1812-1822.

50. Gill, M.R., Reiley, D.G. and Green, S.M. (2004). Interrater reliability of Glasgow Coma Scale seores in the emergency department. Annals of emergency medicine 43, 215-223.

51. Rosenbaum, S.B. and Lipton, M.L. (2012). Embracing chaos: the scope and importance of clinical and pathological heterogeneity in mTBI. Brain imaging and behavior 6, 255-282. 
53. Tebani, A., Abily-Donval, L., Afonso, C., Marret, S. and Bekri, S. (2016). Clinical metabolomics: The new metabolic window for inborn errors of metabolism investigations in the post-genomic era. International Journal of Molecular Sciences 17, 1167. 


\begin{tabular}{|c|c|c|c|c|c|c|c|}
\hline \multirow{2}{*}{ 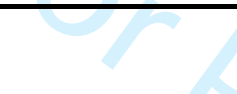 } & & \multicolumn{3}{|c|}{ Discovery cohort (Turku) } & \multicolumn{3}{|c|}{ Validation cohort (Cambridge) } \\
\hline & & Mild TBI & Moderate TBI & Severe TBI & Mild TBI & Moderate TBI & Severe TBI \\
\hline \# of samples & & 108 & 14 & 22 & 36 & 7 & 23 \\
\hline \multirow[t]{3}{*}{ Age } & Mean & 48.37 & 59.57 & 55.05 & 36.75 & 41.57 & 44.87 \\
\hline & $\pm \mathrm{SD}$ & 20.18 & 17.32 & 15.25 & 18.20 & 20.49 & 17.71 \\
\hline & Range & $(18-91)$ & $(32-88)$ & $(19-77)$ & $(16-84)$ & $(19-68)$ & $(20-69)$ \\
\hline Gender & Male / female & $74 / 34$ & $8 / 6$ & $19 / 3$ & $27 / 9$ & $7 / 0$ & $17 / 6$ \\
\hline \multirow[t]{8}{*}{ Mechanism of injury } & ВTH & 4 & 0 & 1 & 5 & 0 & 4 \\
\hline & $\mathrm{A} / \mathrm{C}$ & 18 & 3 & 3 & 8 & 2 & 8 \\
\hline & $\mathrm{V}$ & 11 & 1 & 0 & 0 & 0 & 0 \\
\hline & GLF & 45 & 6 & 10 & 10 & 1 & 1 \\
\hline & FFH & 23 & 3 & 6 & 6 & 3 & 4 \\
\hline & HAO & 5 & 1 & 0 & 5 & 0 & 6 \\
\hline & $\mathrm{O}$ & 0 & 0 & 1 & 2 & 1 & 0 \\
\hline & N/A & 2 & 0 & 1 & 1 & 0 & 0 \\
\hline \multirow[t]{2}{*}{ GCS } & Total (mean) & 14.19 & 9.77 & 4.44 & 14.54 & 10.44 & 5.68 \\
\hline & Motor score (mean) & 5.55 & 4.15 & 1.33 & 5.66 & 4.89 & 3.09 \\
\hline \multirow[t]{4}{*}{ Pupillary reactions } & Both present & 103 & 10 & 12 & 29 & 5 & 12 \\
\hline & Either sluggish & 2 & 3 & 0 & 0 & 2 & 4 \\
\hline & Either absent & 0 & 1 & 8 & 1 & 0 & 5 \\
\hline & N/A & 3 & 0 & 2 & 6 & 0 & 2 \\
\hline CT negative & No visual pathology (Marshall 1) & 57 & 4 & 3 & 28 & 1 & 2 \\
\hline \multirow[t]{5}{*}{ CT positive } & Diffuse injury 2 (Marshall 2) & 28 & 0 & 1 & 4 & 1 & 9 \\
\hline & Diffuse injury 3 (Marshall 3) & 0 & 0 & 2 & 0 & 0 & 0 \\
\hline & Diffuse injury 4 (Marshall 4) & 0 & 0 & 0 & 0 & 0 & 0 \\
\hline & Evacuated mass lesion (Marshall 5) & 8 & 5 & 14 & 0 & 1 & 4 \\
\hline & Non-evacuated mass lesion (Marshall 6) & 15 & 5 & 2 & 4 & 4 & 8 \\
\hline Patient trajectory & Discharged / Admitted to hospital & $42 / 66$ & $0 / 14$ & $0 / 22$ & $27 / 9$ & $0 / 7$ & $0 / 23$ \\
\hline
\end{tabular}

Mechanism of injury: BTH, blow to head; A/C, acceleration/deceleration; V, violence; GLF, ground level fall; FFH, fall from height; HAO; head against object; O, other; N/A, not available. 
Table 2: Metabolites that increased or decreased in concentration when comparing TBI patients who had

visible CT injury compared to CT negative patients (Aim 1). Metabolites in bold were included in the

logistic regression model.

\begin{tabular}{|c|c|c|c|c|c|c|c|c|c|c|c|c|c|}
\hline \multirow[b]{2}{*}{ ID } & \multirow[b]{2}{*}{ Name } & \multirow[b]{2}{*}{ RI } & \multicolumn{2}{|c|}{$\begin{array}{l}\text { CT Negative } \\
\text { Turku }\end{array}$} & \multicolumn{4}{|c|}{ CT Positive Turku } & \multicolumn{2}{|c|}{$\begin{array}{l}\text { CT Negative } \\
\text { Cambridge }\end{array}$} & \multicolumn{3}{|c|}{ CT Positive Cambridge } \\
\hline & & & Mean & SEM & Mean & SEM & Fold & q value & Mean & SEM & Mean & SEM & Fold \\
\hline 1146 & d-Galactose & 1983.12 & 5.91 & 0.18 & 7.27 & 0.23 & 1.23 & 0.0012 & 4.02 & 0.44 & 6.18 & 0.52 & 1.54 \\
\hline 3274 & $\begin{array}{c}\text { Unknown sugar } \\
\text { derivative }\end{array}$ & 2001.78 & 1.87 & 0.12 & 3.07 & 0.21 & 1.64 & 0.0012 & 2.04 & 0.19 & 2.83 & 0.36 & 1.39 \\
\hline 52 & A203003 (sugar) & 2084.20 & 7.15 & 0.05 & 7.48 & 0.05 & 1.05 & 0.0012 & 6.96 & 0.05 & 7.29 & 0.06 & 1.05 \\
\hline 3288 & 3-Deoxyhexitol & 1917.94 & 0.71 & 0.06 & 1.56 & 0.18 & 2.21 & 0.0028 & 0.61 & 0.00 & 1.36 & 0.24 & 2.23 \\
\hline 268 & Phenolic compound & 1274.12 & 3.16 & 0.10 & 2.36 & 0.15 & 0.75 & 0.0028 & 1.75 & 0.25 & 1.08 & 0.22 & 0.62 \\
\hline 208 & $\begin{array}{l}\text { Unknown sugar ( } \\
\text { saccharide) }\end{array}$ & 2364.79 & 3.00 & 0.18 & 4.03 & 0.17 & 1.34 & 0.0028 & 2.29 & 0.29 & 3.30 & 0.33 & 1.45 \\
\hline 428 & 2-Aminobutyric acid & 1186.62 & 3.64 & 0.17 & 2.81 & 0.15 & 0.77 & 0.0081 & 2.89 & 0.29 & 2.42 & 0.20 & 0.84 \\
\hline 104 & Glutamic acid & 1547.38 & 4.73 & 0.07 & 5.10 & 0.07 & 1.08 & 0.0081 & 4.59 & 0.21 & 4.93 & 0.14 & 1.07 \\
\hline 3266 & Amino acid & 1723.54 & 1.43 & 0.05 & 2.04 & 0.14 & 1.43 & 0.0081 & 1.71 & 0.20 & 4.98 & 0.25 & 2.91 \\
\hline 398 & 3-Deoxyhexonic acid & 1917.13 & 2.97 & 0.20 & 1.94 & 0.19 & 0.65 & 0.0081 & 1.00 & 0.32 & 0.50 & 0.20 & 0.50 \\
\hline 1342 & 3-Oxobutanoic acid & 1139.57 & 1.86 & 0.09 & 2.65 & 0.19 & 1.42 & 0.0153 & 3.66 & 0.38 & 4.20 & 0.41 & 1.15 \\
\hline 1348 & Acetoacetic acid & 1261.54 & 0.41 & 0.07 & 1.03 & 0.15 & 2.50 & 0.0172 & 1.68 & 0.27 & 2.22 & 0.36 & 1.32 \\
\hline 50 & Glycerol-3-phosphate & 1806.15 & 5.93 & 0.05 & 5.68 & 0.05 & 0.96 & 0.0178 & 5.97 & 0.09 & 5.34 & 0.08 & 0.89 \\
\hline 65 & Arabinofuranose & 1648.13 & 2.62 & 0.06 & 2.92 & 0.06 & 1.11 & 0.0178 & 2.44 & 0.10 & 2.39 & 0.13 & 0.98 \\
\hline 1365 & $\begin{array}{l}\text { A190025 (hydroxy } \\
\text { acid) }\end{array}$ & 2035.70 & 2.22 & 0.18 & 3.27 & 0.25 & 1.47 & 0.0178 & 1.85 & 0.20 & 2.61 & 0.34 & 1.41 \\
\hline 3279 & Amino acid & 2556.36 & 0.87 & 0.07 & 1.36 & 0.12 & 1.57 & 0.0178 & 0.86 & 0.06 & 1.35 & 0.16 & 1.56 \\
\hline 14 & 3-Hydroxybutyric acid & 1177.70 & 6.10 & 0.14 & 6.71 & 0.12 & 1.10 & 0.0184 & 6.02 & 0.22 & 6.35 & 0.21 & 1.05 \\
\hline 131 & $\begin{array}{c}\text { Phosphoric } \\
\text { acidmonomethylester }\end{array}$ & 1198.29 & 5.41 & 0.05 & 5.03 & 0.10 & 0.93 & 0.0184 & 5.74 & 0.09 & 5.34 & 0.10 & 0.93 \\
\hline 51 & Aminomalonic acid & 1488.39 & 5.28 & 0.08 & 4.92 & 0.07 & 0.93 & 0.0184 & 6.22 & 0.08 & 5.57 & 0.17 & 0.90 \\
\hline 202 & $\begin{array}{c}2- \\
\text { Monopalmitoylglycerol }\end{array}$ & 2563.81 & 3.15 & 0.04 & 3.31 & 0.04 & 1.05 & 0.0184 & 0.58 & 0.08 & 1.43 & 0.22 & 2.44 \\
\hline 165 & Octanoic acid & 1273.01 & 4.11 & 0.17 & 4.96 & 0.20 & 1.21 & 0.0256 & 3.76 & 0.26 & 5.70 & 0.24 & 1.52 \\
\hline 179 & Unknown amino acid & 1380.72 & 3.30 & 0.13 & 2.77 & 0.13 & 0.84 & 0.0393 & 3.49 & 0.17 & 3.10 & 0.20 & 0.89 \\
\hline 253 & Pentitol, 3-desoxy & 1659.63 & 1.27 & 0.09 & 1.61 & 0.08 & 1.27 & 0.0450 & -0.32 & 0.04 & 0.27 & 0.17 & -0.85 \\
\hline 112 & Amino Acid & 1272.83 & 6.45 & 0.06 & 6.24 & 0.05 & 0.97 & 0.0450 & 6.35 & 0.07 & 5.92 & 0.16 & 0.93 \\
\hline 3258 & Phenolic compound & 1652.74 & 0.45 & 0.13 & 1.18 & 0.20 & 2.62 & 0.0450 & 0.39 & 0.22 & 3.13 & 0.25 & 7.97 \\
\hline 183 & $\begin{array}{l}\text { 1H-Indole-3-acetic } \\
\text { acid, 1-hydroxy }\end{array}$ & 1984.84 & 2.82 & 0.10 & 2.30 & 0.15 & 0.82 & 0.0459 & 2.83 & 0.13 & 1.88 & 0.20 & 0.67 \\
\hline 577 & Tartronic acid & 1417.60 & 1.49 & 0.09 & 1.18 & 0.06 & 0.79 & 0.0459 & 0.98 & 0.05 & 1.15 & 0.11 & 1.17 \\
\hline 1271 & $\begin{array}{l}\text { Unknown sugar } \\
\text { (deoxyaldose) }\end{array}$ & 2397.39 & 3.64 & 0.19 & 4.34 & 0.16 & 1.19 & 0.0459 & 2.24 & 0.30 & 3.32 & 0.36 & 1.48 \\
\hline 340 & Serotonin & 2484.34 & 1.07 & 0.13 & 0.61 & 0.11 & 0.57 & 0.0459 & 1.69 & 0.30 & 0.76 & 0.23 & 0.45 \\
\hline 546 & $\begin{array}{l}\text { A249004 (sugar, } \\
\text { saccharide) }\end{array}$ & 2402.27 & 2.20 & 0.18 & 1.57 & 0.14 & 0.71 & 0.0472 & 1.11 & 0.14 & 1.35 & 0.19 & 1.22 \\
\hline 99 & Decanoic acid & 1464.27 & 3.80 & 0.14 & 4.44 & 0.18 & 1.17 & 0.0472 & 3.80 & 0.21 & 5.26 & 0.20 & 1.38 \\
\hline 103 & Diethylene glycol & 1260.24 & 4.08 & 0.06 & 4.28 & 0.04 & 1.05 & 0.0472 & 3.96 & 0.04 & 3.99 & 0.04 & 1.01 \\
\hline 113 & Inositol & 2075.09 & 4.10 & 0.09 & 4.45 & 0.09 & 1.09 & 0.0472 & 3.68 & 0.25 & 4.20 & 0.24 & 1.14 \\
\hline 1237 & Riboinic acid & 1796.93 & 1.61 & 0.10 & 1.98 & 0.09 & 1.23 & 0.0484 & 1.54 & 0.19 & 2.05 & 0.17 & 1.33 \\
\hline 178 & Glucuronic acid & 1987.55 & 5.02 & 0.14 & 4.47 & 0.14 & 0.89 & 0.0484 & 3.97 & 0.22 & 4.27 & 0.22 & 1.08 \\
\hline 1330 & $\begin{array}{c}\text { Unknown carboxylic } \\
\text { acid }\end{array}$ & 1443.76 & 1.57 & 0.12 & 2.12 & 0.16 & 1.35 & 0.0495 & 2.14 & 0.23 & 2.56 & 0.24 & 1.20 \\
\hline
\end{tabular}


Table 3: Metabolites that increased or decreased in concentration when comparing TBI patients who had a diffuse injury compared to a mass lesion as observed by a CT scan (Aim 2). Metabolites in bold were included in the logistic regression model.

\begin{tabular}{|c|c|c|c|c|c|c|c|c|c|c|c|c|c|}
\hline \multirow[b]{2}{*}{ ID } & \multirow{2}{*}{ Name } & \multirow[b]{2}{*}{ RI } & \multicolumn{2}{|c|}{$\begin{array}{l}\text { Diffuse } \\
\text { injury Turku }\end{array}$} & \multicolumn{4}{|c|}{ Mass lesion Turku } & \multicolumn{2}{|c|}{$\begin{array}{c}\text { Diffuse } \\
\text { injury } \\
\text { Cambridge }\end{array}$} & \multicolumn{3}{|c|}{$\begin{array}{l}\text { Mass lesion } \\
\text { Cambridge }\end{array}$} \\
\hline & & & Mean & SEM & Mean & SEM & Fold & q value & Mean & SEM & Mean & SEM & Fold \\
\hline 15 & 2-Hydroxybutyric acid & 1146.93 & 5.93 & 0.07 & 6.35 & 0.08 & 1.07 & 0.02 & 5.30 & 0.13 & 5.67 & 0.14 & 1.07 \\
\hline 167 & Isovalerylglucuronide & 2033.50 & 5.14 & 0.33 & 6.22 & 0.08 & 1.21 & 0.02 & 4.34 & 0.66 & 5.20 & 0.53 & 1.20 \\
\hline 99 & Decanoic acid & 1464.27 & 3.71 & 0.24 & 4.91 & 0.23 & 1.32 & 0.03 & 4.84 & 0.32 & 5.54 & 0.25 & 1.14 \\
\hline 183 & $\begin{array}{c}\text { 1H-Indole-3-acetic acid, } \\
\text { 1-hydroxy }\end{array}$ & 1984.84 & 2.90 & 0.13 & 1.93 & 0.21 & 0.66 & 0.03 & 2.10 & 0.33 & 1.73 & 0.27 & 0.82 \\
\hline 3258 & Phenolic compound & 1652.74 & 0.39 & 0.20 & 1.69 & 0.28 & 4.33 & 0.05 & 2.15 & 0.49 & 3.78 & 0.09 & 1.76 \\
\hline
\end{tabular}




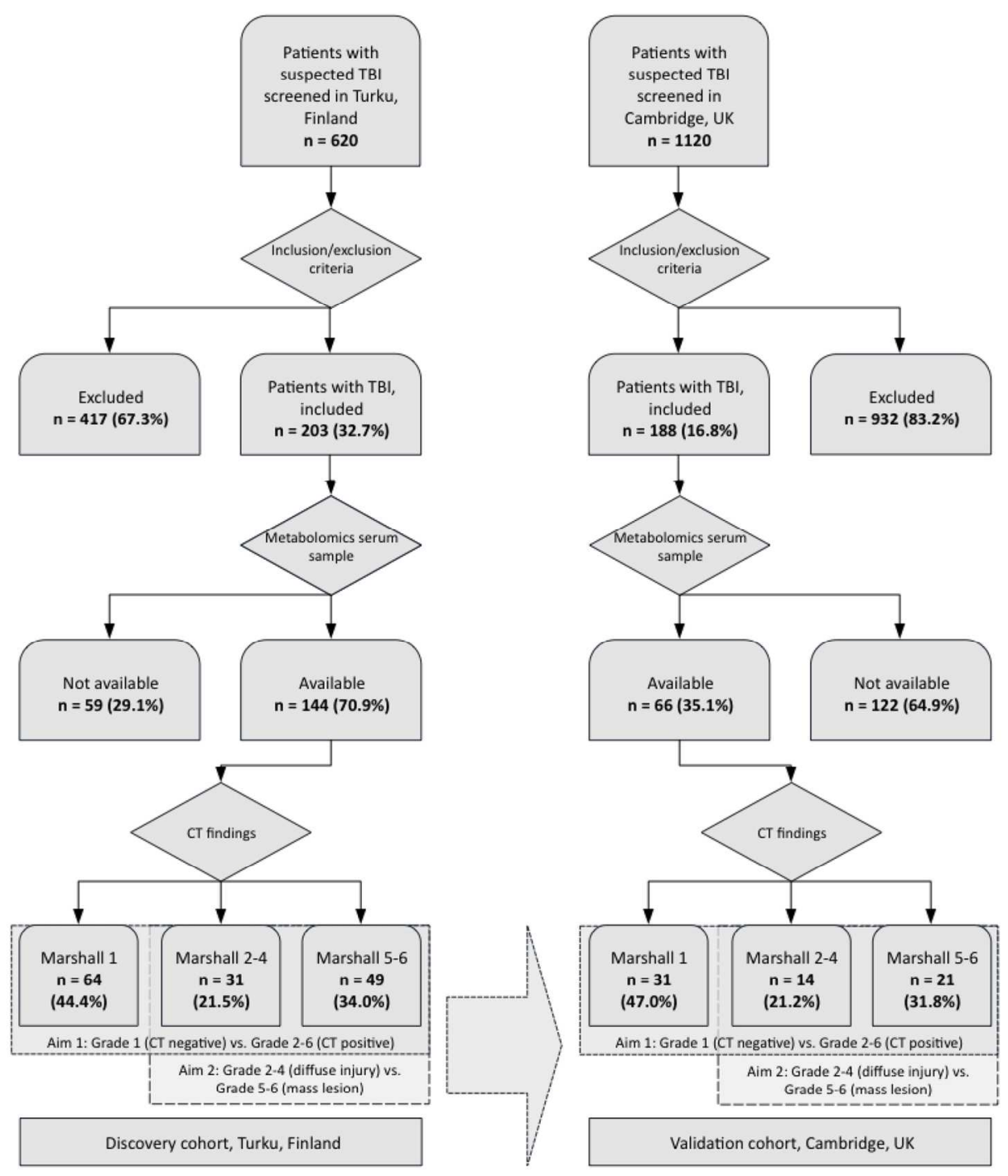

Figure 1: Flow chart showing the patient selection process.

$141 \times 161 \mathrm{~mm}(300 \times 300 \mathrm{DPI})$ 


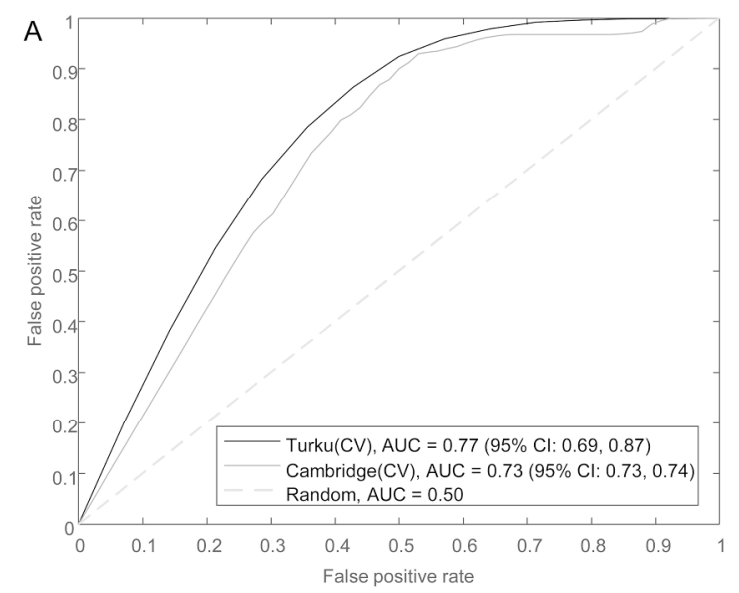

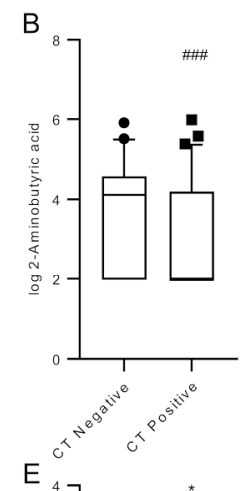

C

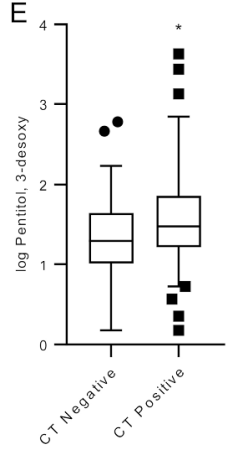

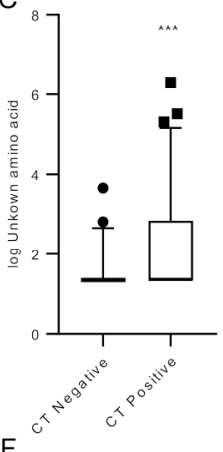

$F$

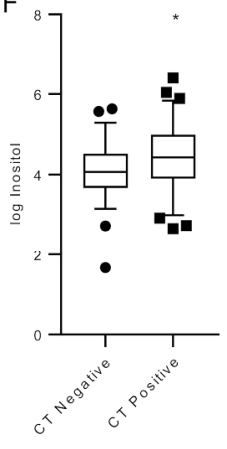

D

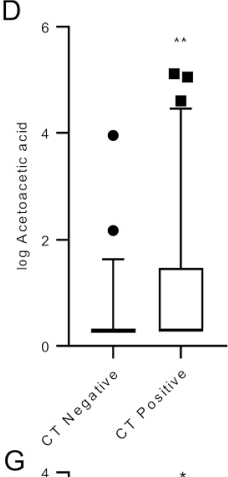

G

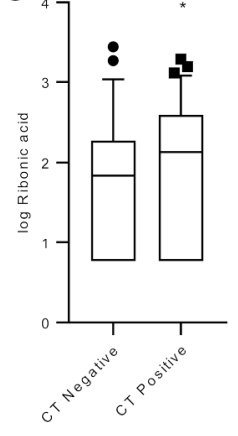

Figure 2: Serum metabolites can predict the need for a CT scan following TBI. (A) The ROC curve showing the performance characteristics of the logistic regression model for both the discovery cohort (Turku) and the validation cohort (Cambridge). (B-G) Box and whisker plots of the metabolites used to generate the logistic regression model (A). For univariate stats see table 2. Key: AUC - area under the curve, ROC receiver operator curve. The boxes show mean and SEM with the whiskers extending to the $95 \%$ percentile. Outliers are marked as squares (Turku) or circles (Cambridge).

$$
\text { 202x410mm (300 x } 300 \text { DPI) }
$$



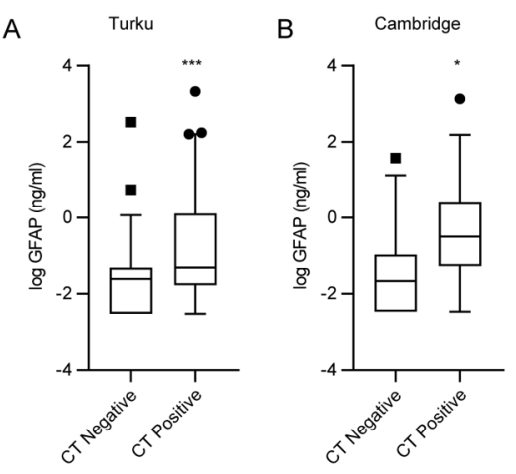

C

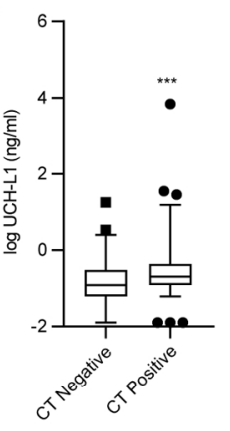

D

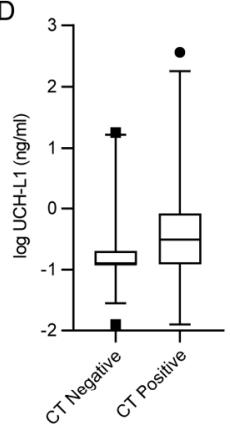

E

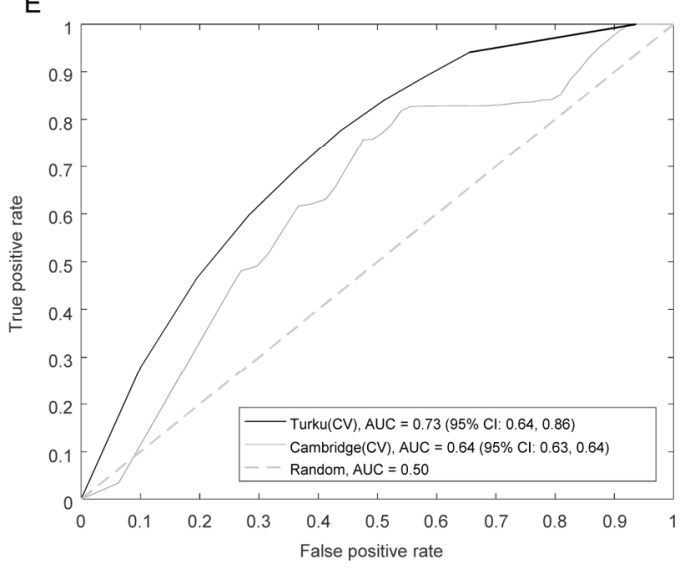

Figure 3: GFAP and UCH-L1 concentrations increase following TBI. (A-D) Box and whisker plots of the log scaled GFAP ( $A$ and $B$ ) and UCH-L1 (C and D) concentrations in both the Turku ( $A$ to $C$ ) and Cambridge cohorts ( $B$ to $D)$. (E) The ROC curve showing the performance characteristics of the logistic regression model for both the discovery cohort (Turku) and the validation cohort (Cambridge). Key: the boxes show mean and SEM with the whiskers extending to the 95\% percentile. Outliers are marked as squares (Turku) or circles (Cambridge), AUC - area under the curve, ROC - receiver operator curve.

$84 \times 187 \mathrm{~mm}(300 \times 300 \mathrm{DPI})$ 

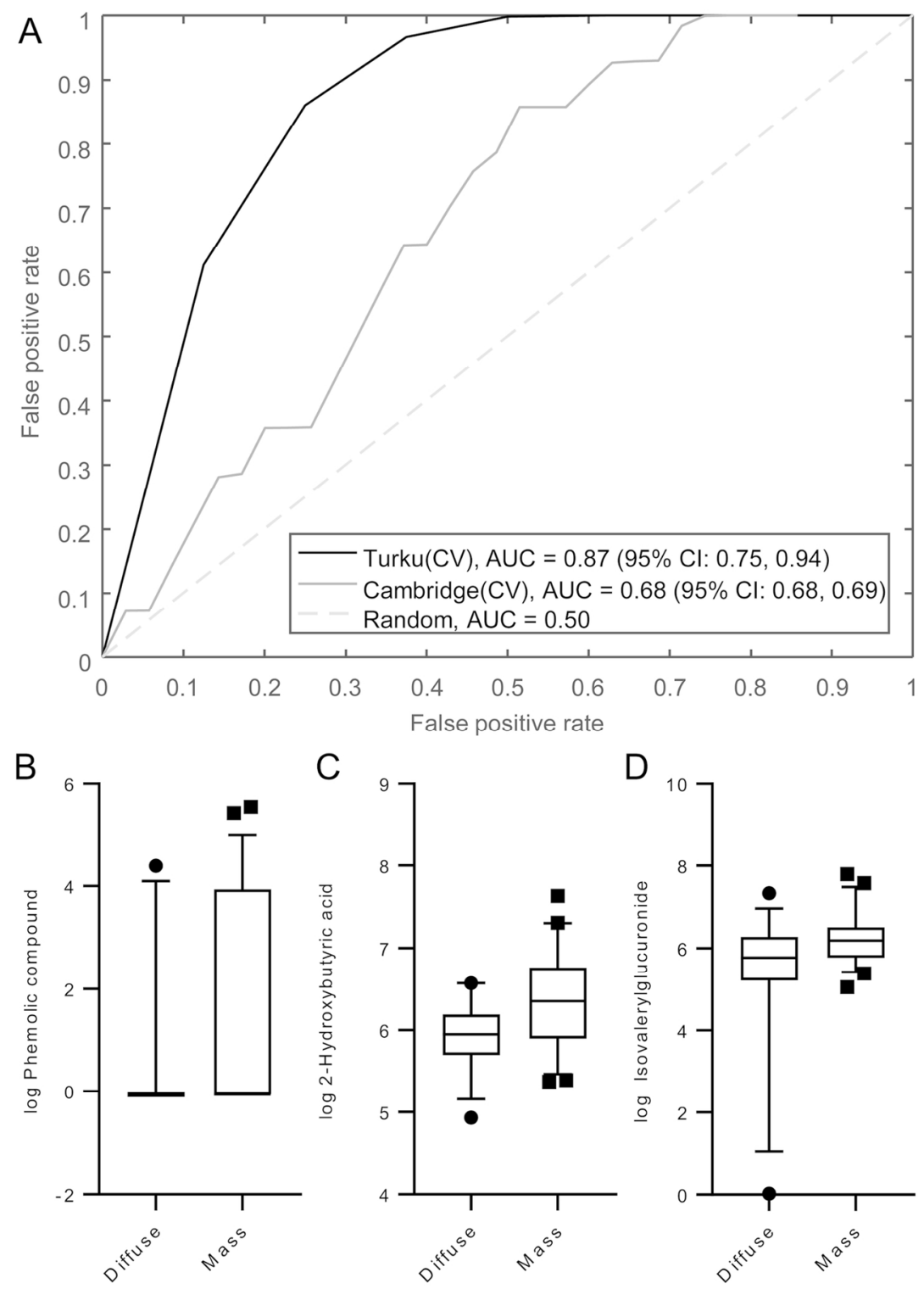

Figure 4: Serum metabolites can discriminate between diffuse injury and mass lesions as observed in a CT scan. (A) The ROC curve showing the performance characteristics of the logistic regression model for both the discovery cohort (Turku) and the validation cohort (Cambridge). (B-D) Box and whisker plots of the metabolites used to generate the logistic regression model (A). For univariate stats see table 3. Key: AUC area under the curve, ROC - receiver operator curve. The boxes show mean and SEM with the whiskers extending to the $95 \%$ percentile. Outliers are marked as squares (Turku) or circles (Cambridge).

$$
133 \times 179 \mathrm{~mm}(300 \times 300 \mathrm{DPI})
$$




\section{A $\quad \log _{2}([$ Serum(sTBI) $] /[$ Serum(Control) $])$}
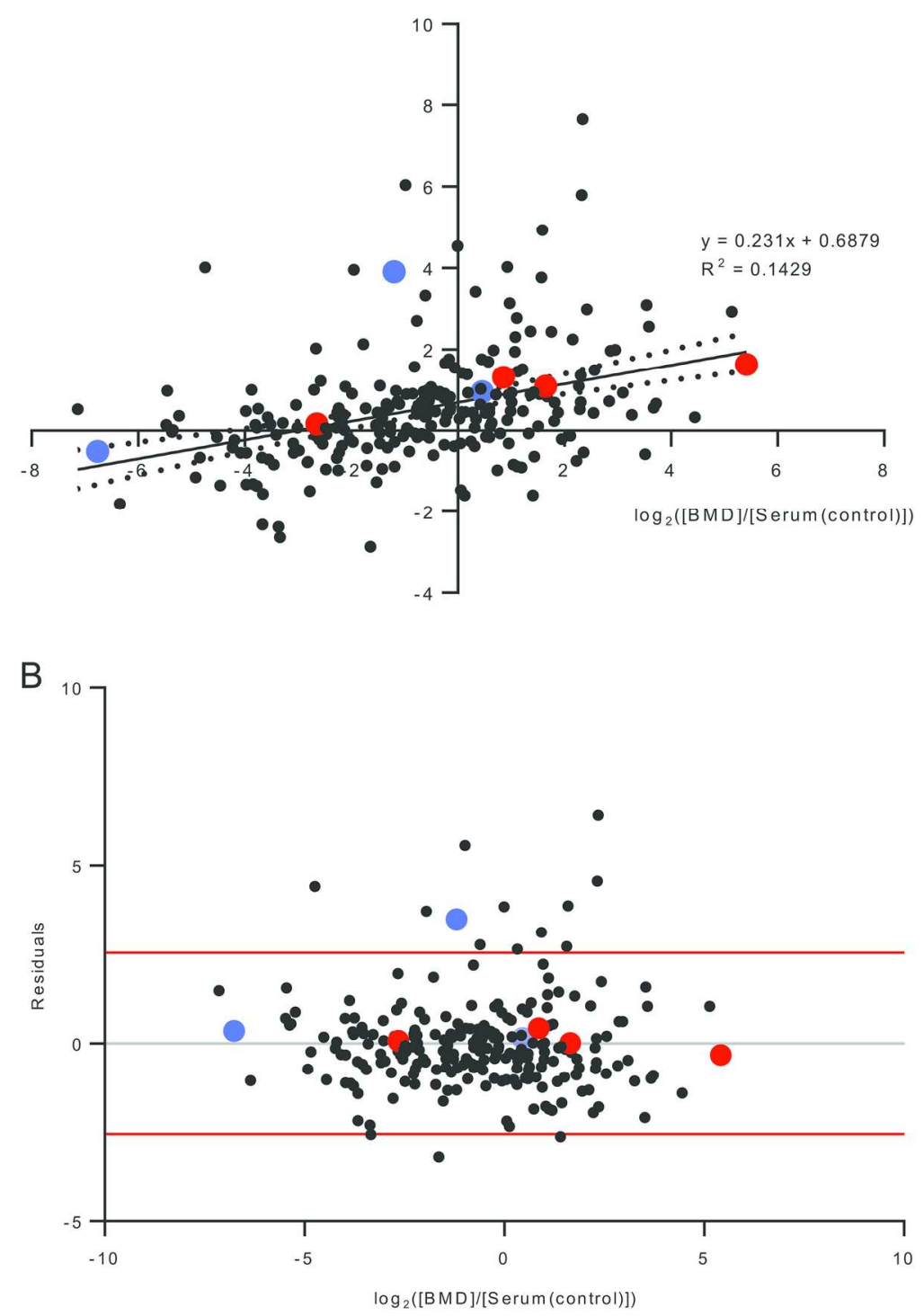

Figure 5: Associations of serum metabolites identified in the logistic regression modelling with their concentrations in the brain microdialysates (BMD). (A) Reproduction of the comparison of mean serum metabolite level changes (severe TBI vs. controls) and mean BMD metabolite levels (BMD vs. serum controls)22. The regression line was calculated using all data points. (B) Scatter graph showing the distance between the predicted and the actual Y value (residual) for each metabolite plotted in (A). Key: Red circles metabolites that predicted the need for a CT scan; Blue circles - metabolites that differentiated diffuse injury compared to a mass lesion; Dotted lines - 95\% confidence interval of model; Grey line highlights the origin Red lines - Two time standard deviation of the residuals. 


\section{Figure Legends}

\section{Figure 1: Flow chart showing the patient selection process.}

Figure 2: Serum metabolites can predict the need for a CT scan following TBI. (A) The ROC curve showing the performance characteristics of the logistic regression model for both the discovery cohort (Turku) and the validation cohort (Cambridge). (B-G) Box and whisker plots of the metabolites used to generate the logistic regression model (A). For univariate stats see table 2. Key: AUC - area under the curve, ROC - receiver operator curve. The boxes show mean and SEM with the whiskers extending to the $95 \%$ percentile. Outliers are marked as squares (Turku) or circles (Cambridge).

Figure 3: GFAP and UCH-L1 concentrations increase following TBI. (A-D) Box and whisker plots of the log scaled GFAP (A and B) and UCH-L1 (C and D) concentrations in both the Turku (A to C) and Cambridge cohorts (B to D). (E) The ROC curve showing the performance characteristics of the logistic regression model for both the discovery cohort (Turku) and the validation cohort (Cambridge). Key: the boxes show mean and SEM with the whiskers extending to the $95 \%$ percentile. Outliers are marked as squares (Turku) or circles (Cambridge), AUC - area under the curve, ROC - receiver operator curve.

Figure 4: Serum metabolites can discriminate between diffuse injury and mass lesions as observed in a CT scan. (A) The ROC curve showing the performance characteristics of the logistic regression model for both the discovery cohort (Turku) and the validation cohort (Cambridge). (B-D) Box and whisker plots of the metabolites used to generate the logistic regression model (A). For univariate stats see table 3. Key: AUC - area under the curve, ROC - receiver operator curve. The boxes show mean and SEM with the whiskers extending to the 95\% percentile. Outliers are marked as squares (Turku) or circles (Cambridge). 


\section{Figure 5: Associations of serum metabolites identified in the logistic regression modelling with their concentrations in the brain microdialysates (BMD). (A)} Reproduction of the comparison of mean serum metabolite level changes (severe TBI vs. controls) and mean BMD metabolite levels (BMD vs. serum controls) ${ }^{22}$. The regression line was calculated using all data points. (B) Scatter graph showing the distance between the predicted and the actual Y value (residual) for each metabolite plotted in (A). Key: Red circles - metabolites that predicted the need for a CT scan; Blue circles - metabolites that differentiated diffuse injury compared to a mass lesion; Dotted lines - 95\% confidence interval of model; Grey line highlights the origin Red lines - Two time standard deviation of the residuals. 

metabolomics biomarkers (or least the classes) need to be introduced, particularly the ones being examined in the study. Specify which metabolites have been correlated with lesions on CT before.

Given the lack of studies in metabolites predicting the need for a CT scan it is tricky to expand these parts but we have added the following sentences into the introduction:

"To date the application of modern untargeted metabolomic approaches to study TBI have been sparse and to our knowledge there are no studies exploring the role of metabolomics in detecting the need for a CT scan following a TBI.

This study will use gas chromatography which will identify small polar metabolites such as sugars, amino acids and free fatty acids in an untargeted approach $(28,29)$ to screen for new metabolites which can predict the need for a CT following a TBI."

- The data on the number of the patients who were screened (Figure 1) should go in the results and NOT in the methods.

We have moved Figure 1 to the results as suggested. 
- In the methods describe that rationale behind the discovery the validation cohorts and present the results accordingly.

The rationale behind the discovery and validation sets are outlined in the introduction. Without the two independent groups it is possible to overfit the data has often been seen in the protein literature.

We have added the following sentence to the methods to clarify this point:

'The need for a discovery and validation cohorts prevents the over modelling of the data often seen in multivariate statistics.'

We have also added in the discovery and validation identifiers into the results.

- Clearly define negative and positive CT.

To clarify the descriptions of CT positive and CT Negative we have added the word Marshall into the appropriate section in the methods where the groups were defined. It now reads:

Aim 1: Marshall Grade 1 (CT negative) vs. Marshall Grade 2-6 (CT positive)

Aim 2: Marshall Grade 2-4 (diffuse injury) vs. Marshall Grade 5-6 (mass lesion)

- Sometimes the authors keep separate the results from the 2 sites and sometimes they use them together and it makes the results confusing.

We are unsure how to respond to this comment as in the results we always use the Turku data as a discovery group and the Cambridge as a validation group. We never mix the two sites.

- Data analysis has to follow the objectives so describe the analyses relative to the objectives. Please describe the statistic used for each objective.

We have added the names of the univariate statistic test used to identify the significant metabolites separating each group in order to clarify the statistics for the reader.

- What were the dates of recruitment? What was the timing the samples collected relative to time of injury?

All of the samples have been obtained within 12 hours of hospital admission. There are some patients who have been found unconscious and transferred to the centers with latency - in these cases, an exact time of the injury is unknown. Additionally, there are patients who have sustained mild TBI and sought for medical attention with latency as well. Both of these situations reflect the reality in clinical practice. This has now been mentioned in the manuscript.

- Table 1 should be transposed (pivot) so the horizontal indices are vertical and vice versa. Add mechanism of injury, pupillary response, GCS Motor score, and if they were admitted or discharged, number of negative vs positive CT's.

We have updated Table 1 as requested by the reviewer.

- In Table 2 Can the authors place the metabolites in some kind of classification scheme rather than just listing them?

The logistic regression models provide this classification scheme and for full disclosure we would like to publish the fold changes and univariate results. In theory these tables could be placed as supplemental information if required.

- A sensitivity of 0.73 and specificity of 0.64 is not very strong. This needs to be discussed further.

We have added the following sentence to the discussion: 
"Additionally, the fact that this was a global metabolite screen could contribute to the lower sensitivity and specificity observed in the metabolite model. A targeted screen should help increase this sensitivity and specificity. However, it is worth noting that the metabolites did outperform the proteins when employed in a multi-center setting which is more clinically relevant."

- The results of the modelling should be shown but placed in an appendix.

The results of the modelling are the key part of this paper given we have now removed two of the aims and the MTBI results we think the results are now clear enough for the reader.

- Page 20 Line 44: the statement "...thus suggesting that the metabolites are more reliable in a multicenter setting as compared to proteins, when predicting the need for $\mathrm{CT}^{\prime \prime}$ cannot be said based on the data (inaccurate statement) and no opinions should be voiced in the results.

We disagree with the reviewer on this point, this study clearly demonstrates that the AUC obtained from metabolomics outperform GFAP, UCHL-1 or a combination of both when performed in a multicenter setting. To illustrate this point we have included an additional ROC plot highlighting the difference between the protein and metabolomics results. This is now Figure $3 \mathrm{~A}$ We agree that this statement was in the incorrect place and have removed from the results.

- Marshall Class does not apply well to mild TBI and does not provide a good grading of severity in these patients. Provide a justification in the methods for using this grading system even though it has no role in mild TBI patients.

We acknowledge that Marshall classification is not suitable for severity grading - especially in terms of mild TBI. However, in the current study, we focus on the imaging findings seen on the first CT. For this purpose Marshall classification is appropriate as we wanted to analyze patients with negative CT findings (Marshall Grade 1) and positive CT findings (Marshall Grade 2-6) and patients with diffuse injury (Marshall Grade 2-4) and mass lesions (Marshall Grade 5-6). A short comment on the justification of use of the scale is now added.

- Who read the CT scans? What was the inter-rater reliability of the radiologists?

The CT scans were analyzed by neuroradiologists and double-read by a senior neurosurgeron (JPP) and a neurologist (OT). This has now been stated in the manuscript. There were no disagreements on the imaging findings.

- Provide a sample size calculation to justify that there were enough patients to make a valid conclusion. There are so many comparisons for the number of patients that the authors need to justify they have enough patients.

- There are not enough patients to model mild TBI patients alone.

We have removed the mTBI results from this paper.

- The study does not demonstrate the superiority of metabolomics at assessing the need for a CT.

We disagree with this comment. The AUC for the metabolomics in the validation cohort is better than the protein data over 1000 models. As stated in the introduction the majority of the protein results in this area do not have a proper discovery validation set-up and thus are prone to overfitting. This is an important point to this paper.

- There has to be some logic in why these particular metabolites were predictive. The fact that there are still significant differences in AUC's between the sites really questions the reliability of the group of markers selected.

The fact that AUCs between the sites are not the same cannot be considered as unusual or as weakness of the study. From the model testing perspective, the two study sites cannot possibly be considered as equivalent. One was used as a discovery study, i.e. where the metabolite-based model was developed, 
while the other was a validation study, where the model was independently tested and thus avoiding the potential issue of overfitting. It is typical that the models perform better in the discovery studies because that's where the models are optimized. However, the fact is that in both reported studies, discovery and validation, significant effects have been observed including in terms of diagnostic performance. 